\title{
Uniform boundary estimates in homogenization of higher-order elliptic systems
}

\author{
Weisheng $\mathrm{Niu}^{1}$ (D) Y Yao $\mathrm{Xu}^{2}$
}

Received: 2 February 2018 / Accepted: 22 May 2018 / Published online: 2 June 2018

(C) Fondazione Annali di Matematica Pura ed Applicata and Springer-Verlag GmbH Germany, part of Springer Nature 2018

\begin{abstract}
This paper focuses on uniform boundary estimates in homogenization of a family of higher-order elliptic operators $\mathcal{L}_{\varepsilon}$, with rapidly oscillating periodic coefficients. We derive uniform boundary $C^{m-1, \lambda}(0<\lambda<1)$ and $W^{m, p}$ estimates in $C^{1}$ domains, as well as uniform boundary $C^{m-1,1}$ estimate in $C^{1, \theta}(0<\theta<1)$ domains without the symmetry assumption on the operator. The proof, motivated by the works "Armstrong and Smart in Ann Sci Éc Norm Supér (4) 49(2):423-481 (2016) and Shen in Anal PDE 8(7):1565-1601 (2015)," is based on a suboptimal convergence rate in $H^{m-1}(\Omega)$. Compared to "Kenig et al. in Arch Ration Mech Anal 203(3):1009-1036 (2012) and Shen (2015)," the convergence rate obtained here does not require the symmetry assumption on the operator, nor additional assumptions on the regularity of $u_{0}$ (the solution to the homogenized problem), and thus might be of some independent interests even for second-order elliptic systems.
\end{abstract}

Keywords Elliptic systems · Homogenization · Uniform estimates · Convergence rate

Mathematics Subject Classification 35B27 · 35J48

Supported in part by the NSF of China (11701002) and Anhui Province (1708085MA02).

Weisheng Niu

niuwsh@ahu.edu.cn

Yao Xu

dg1421012@smail.nju.edu.cn

1 School of Mathematical Science, Anhui University, Hefei 230601, People's Republic of China

2 Department of Mathematics, Nanjing University, Nanjing 210093, People's Republic of China 


\section{Introduction}

This paper is aimed to investigate the uniform boundary estimates in homogenization of the following $2 m$-order elliptic system,

$$
\begin{cases}\mathcal{L}_{\varepsilon} u_{\varepsilon}=f & \text { in } \Omega, \\ \operatorname{Tr}\left(D^{\gamma} u_{\varepsilon}\right)=g_{\gamma} & \text { on } \partial \Omega \text { for } 0 \leq|\gamma| \leq m-1,\end{cases}
$$

where $\Omega \subset \mathbb{R}^{d}, d \geq 1$, is a bounded Lipschitz domain,

$$
\left(\mathcal{L}_{\varepsilon} u_{\varepsilon}\right)_{i}=(-1)^{m} \sum_{|\alpha|=|\beta|=m} D^{\alpha}\left(A_{i j}^{\alpha \beta}(x / \varepsilon) D^{\beta} u_{\varepsilon j}\right), \quad 1 \leq i, j \leq n,
$$

$u_{\varepsilon j}$ denotes the $j$ th component of the $\mathbb{R}^{n}$-valued vector function $u_{\varepsilon}, \alpha, \beta, \gamma$ are multi-indexes with components $\alpha_{k}, \beta_{k}, \gamma_{k}, k=1,2, \ldots, d$, and

$$
|\alpha|=\sum_{k=1}^{d} \alpha_{k}, \quad D^{\alpha}=D_{x_{1}}^{\alpha_{1}} D_{x_{2}}^{\alpha_{2}} \cdots D_{x_{d}}^{\alpha_{d}} .
$$

The coefficients matrix $A(y)=\left(A_{i j}^{\alpha \beta}(y)\right), 1 \leq i, j \leq n$, is real, bounded measurable, satisfying the strong ellipticity condition

$$
\mu|\xi|^{2} \leq \sum_{|\alpha|=|\beta|=m} A_{i j}^{\alpha \beta}(y) \xi_{\alpha}^{i} \xi_{\beta}^{j} \leq \frac{1}{\mu}|\xi|^{2} \text { for a.e. } y \in \mathbb{R}^{d},
$$

where $\mu>0, \xi=\left(\xi_{\alpha}\right)_{|\alpha|=m}, \xi_{\alpha}=\left(\xi_{\alpha}^{1}, \ldots, \xi_{\alpha}^{n}\right) \in \mathbb{R}^{n}$, as well as the periodicity condition

$$
A(y+z)=A(y), \quad \text { for any } z \in \mathbb{Z}^{d} \text { and a.e. } y \in \mathbb{R}^{d} .
$$

The regularity estimate uniform in $\varepsilon>0$ is one of the main concerns in quantitative homogenization. For second-order elliptic operators, this issue has been studied extensively. In the celebrated work of Avellaneda and Lin [5-7], by using a compactness method, the interior and boundary Hölder estimate, $W^{1, p}$ estimate and Lipschitz estimate were obtained for second-order elliptic systems with Hölder continuous coefficients and Dirichlet conditions in bounded $C^{1, \theta}$ domains. The uniform boundary Lipschitz estimate for the Neumann problem has been a longstanding open problem and was recently settled by Kenig et al. [23]. Interested readers may refer to $[20,24,34,36]$ and references therein for more applications of compactness method in quantitative homogenization. More recently, another fabulous scheme, which is based on convergence rates, was formulated in [4] to investigate uniform (interior) estimates in stochastic homogenization. The approach was further developed in $[3,35]$, where the large-scale interior or boundary Lipschitz estimates for second-order elliptic operators with periodic and almost periodic coefficients were studied systematically. We also refer readers to $[2,16,17,46]$ for more related results.

Relatively speaking, few quantitative results were known in the homogenization of higherorder elliptic equations previously, although results on qualitative homogenization have been obtained for many years [9]. Very recently, the optimal $O(\varepsilon)$ convergence rate in $L^{2}\left(\mathbb{R}^{d}\right)$ for higher-order elliptic equations was obtained in $[25,29,30]$. In $[39,40]$, some interesting two-parameter resolvent estimates were established in homogenization of general higherorder elliptic systems with periodic coefficients in bounded $C^{2 m}$ domains. Meanwhile, in $[28,45]$ we investigated the sharp $O(\varepsilon)$ convergence rate in periodic and almost periodic homogenization of higher-order elliptic systems in Lipschitz domains. Particularly, under 
the assumptions that $A$ is symmetric and $u_{0} \in H^{m+1}(\Omega)$, the optimal $O(\varepsilon)$ convergence rate was obtained in $W^{m-1, q_{0}}(\Omega), q_{0}=2 d /(d-1)$ in [28]. Moreover, the uniform interior $W^{m, p}$ and $C^{m-1,1}$ estimates were also established therein.

As a continuation of [28], in this paper we investigate the uniform boundary estimates in homogenization of higher-order elliptic system (1.1). Let $\psi: \mathbb{R}^{d-1} \rightarrow \mathbb{R}$ be a $C^{1}$ function with

$$
\begin{aligned}
& \psi(0)=0, \quad|\nabla \psi| \leq M, \\
& \sup \left\{\left|\nabla \psi\left(x^{\prime}\right)-\nabla \psi\left(y^{\prime}\right)\right|: x^{\prime}, y^{\prime} \in \mathbb{R}^{d-1} \text { and }\left|x^{\prime}-y^{\prime}\right| \leq t\right\} \leq \tau(t),
\end{aligned}
$$

where $\tau(t) \longrightarrow 0$ as $t \longrightarrow 0^{+}$. Set

$$
\begin{aligned}
& D_{r}=D(r, \psi)=\left\{\left(x^{\prime}, x_{d}\right) \in \mathbb{R}^{d}:\left|x^{\prime}\right|<r \text { and } \psi\left(x^{\prime}\right)<x_{d}<\psi\left(x^{\prime}\right)+r\right\}, \\
& \Delta_{r}=\Delta(r, \psi)=\left\{\left(x^{\prime}, \psi\left(x^{\prime}\right)\right) \in \mathbb{R}^{d}:\left|x^{\prime}\right|<r\right\} .
\end{aligned}
$$

The main results of this paper are stated as follows.

Theorem 1.1 Suppose that the coefficient matrix $A=A(y)$ satisfies the conditions (1.2)(1.3) and $u_{\varepsilon} \in H^{m}\left(D_{1} ; \mathbb{R}^{n}\right)$ is a weak solution to

$$
\begin{cases}\mathcal{L}_{\varepsilon} u_{\varepsilon}=F & \text { in } D_{1}, \\ \operatorname{Tr}\left(D^{\gamma} u_{\varepsilon}\right)=D^{\gamma} G & \text { on } \Delta_{1} \text { for } 0 \leq|\gamma| \leq m-1,\end{cases}
$$

where $G \in C^{m-1,1}\left(D_{1} ; \mathbb{R}^{n}\right), F \in L^{p}\left(D_{1} ; \mathbb{R}^{n}\right)$ with $p>\max \{d /(m+1), 2 d /(d+2 m-$ $2), 1\}$. Then, for any $0<\lambda<\min \{m+1-d / p, 1\}$ and any $\varepsilon \leq r<1$,

$$
\left(f_{D_{r}}\left|\nabla^{m} u_{\varepsilon}\right|^{2}\right)^{1 / 2} \leq C r^{\lambda-1}\left\{\left(f_{D_{1}}\left|u_{\varepsilon}\right|^{2}\right)^{1 / 2}+\left(f_{D_{1}}|F|^{p}\right)^{1 / p}+\|G\|_{C^{m-1,1}\left(D_{1}\right)}\right\},
$$

where $C$ depends only on $d, n, m, \lambda, \mu, p$ and $\tau(t)$ in (1.4).

Estimate (1.6) can be viewed as the $C^{m-1, \lambda}$ estimate uniform down to the scale $\varepsilon$ in $C^{1}$ domains for higher-order elliptic operators $\mathcal{L}_{\mathcal{E}}$. In addition to the assumptions in Theorem 1.1, if $A \in V M O\left(\mathbb{R}^{d}\right)$, i.e.,

$$
\sup _{x \in \mathbb{R}^{d}, 0<r<t} f_{B(x, r)}\left|A(y)-f_{B(x, r)} A\right| \mathrm{d} y \leq \varrho(t), \quad 0<t \leq 1,
$$

for some nondecreasing continuous function $\varrho(t)$ on $[0,1]$ with $\varrho(0)=0$, then a standard blow-up argument gives the following full-scale boundary $C^{m-1, \lambda}$ estimate

$$
\left\|u_{\varepsilon}\right\|_{C^{m-1, \lambda}\left(D_{1 / 4}\right)} \leq C\left\{\left(f_{D_{1}}\left|u_{\varepsilon}\right|^{2}\right)^{1 / 2}+\left(f_{D_{1}}|F|^{p}\right)^{1 / p}+\|G\|_{C^{m-1,1}\left(D_{1}\right)}\right\} .
$$

We also mention that the restriction $p>\max \{d /(m+1), 1\}$ is made to ensure $C^{m-1, \lambda}$ estimate of the solution $u_{0}$ to the homogenized system, which plays an essential role in the proof of the theorem. The restriction $p>2 d /(d+2 m-2)$ is used to ensure that $F \in H^{-m+1}(\Omega)$, since our proof is based on the convergence result in Theorem 1.4 (see Lemma 4.1 for details). Although the assumption on the regularity of $F$ in Theorem 1.1 is not sharp, see Corollary 5.1 for the full-scale uniform $C^{m-1, \lambda}$ estimate of $u_{\varepsilon}$, it is enough for us to derive the following uniform $W^{m, p}$ estimate on $u_{\varepsilon}$. 
Theorem 1.2 Let $\Omega$ be a bounded $C^{1}$ domain in $\mathbb{R}^{d}$. Suppose that the coefficient matrix $A \in V M O\left(\mathbb{R}^{d}\right)$ satisfies (1.2)-(1.3) and $u_{\varepsilon} \in H^{m}\left(\Omega ; \mathbb{R}^{n}\right)$ is a weak solution to

$$
\begin{cases}\mathcal{L}_{\varepsilon} u_{\varepsilon}=\sum_{|\alpha| \leq m} D^{\alpha} f^{\alpha} & \text { in } \Omega, \\ \operatorname{Tr}\left(D^{\gamma} u_{\varepsilon}\right)=g_{\gamma} & \text { on } \partial \Omega \text { for } 0 \leq|\gamma| \leq m-1,\end{cases}
$$

where $\dot{g}=\left\{g_{\gamma}\right\}_{|\gamma| \leq m-1} \in \dot{B}_{p}^{m-1 / p}\left(\partial \Omega ; \mathbb{R}^{n}\right)$ and $f^{\alpha} \in L^{p}\left(\Omega ; \mathbb{R}^{n}\right)$ for $|\alpha| \leq m, 2 \leq p<\infty$. Then,

$$
\left\|u_{\varepsilon}\right\|_{W^{m, p}(\Omega)} \leq C_{p}\left\{\sum_{|\alpha| \leq m}\left\|f^{\alpha}\right\|_{L^{p}(\Omega)}+\|\dot{g}\|_{\dot{B}_{p}^{m-1 / p}(\partial \Omega)}\right\},
$$

where the constant $C_{p}$ depends only on $p, d, n, m, \mu, \Omega$ and $\varrho(t)$ in (1.7).

We refer readers to Sect. 2 for the definition of the Whitney-Besov space $\dot{B}_{p}^{s}\left(\partial \Omega ; \mathbb{R}^{n}\right)$. Note that although the result presented in Theorem 1.2 focuses on the case $p \geq 2$, by a standard duality argument, it still holds for $1<p<2$. We also mention that the uniform $W^{1, p}$ estimates in the homogenization of second-order elliptic systems have been studied largely, see e.g., [14, 15,33,43,44]. Theorem 1.2 generalizes the uniform $W^{1, p}$ estimates for second-order elliptic systems to higher-order elliptic systems. It also extends, in some sense, the $W^{m, p}$ estimate for higher-order elliptic equations (or systems) with non-oscillating coefficients, see e.g., [10,12,13].

Our third result gives the uniform boundary $C^{m-1,1}$ estimate of $u_{\varepsilon}$ in $C^{1, \theta}(0<\theta<1)$ domains. Let $D_{r}, \Delta_{r}$ be defined as in (1.5), and let the defining function $\psi \in C^{1, \theta}\left(\mathbb{R}^{d-1}\right)$ with

$$
\psi(0)=0, \quad\|\nabla \psi\|_{C^{\theta}\left(\mathbb{R}^{d-1}\right)} \leq M_{1} .
$$

Theorem 1.3 Assume that A satisfies (1.2)-(1.3). Let $u_{\varepsilon} \in H^{m}\left(D_{1} ; \mathbb{R}^{n}\right)$ be a weak solution to

$$
\begin{cases}\mathcal{L}_{\varepsilon} u_{\varepsilon}=\sum_{|\alpha| \leq m-1} D^{\alpha} f^{\alpha} & \text { in } D_{1}, \\ \operatorname{Tr}\left(D^{\gamma} u_{\varepsilon}\right)=D^{\gamma} G & \text { on } \Delta_{1} \quad \text { for } 0 \leq|\gamma| \leq m-1,\end{cases}
$$

where $f^{\alpha} \in L^{q}\left(D_{1} ; \mathbb{R}^{n}\right)$ with $q>d, q \geq 2$, and $G \in C^{m, \sigma}\left(D_{1} ; \mathbb{R}^{n}\right)$ for some $0<\sigma \leq \theta$. Then, for any $\varepsilon \leq r<1$, we have

$$
\left(f_{D_{r}}\left|\nabla^{m} u_{\varepsilon}\right|^{2}\right)^{1 / 2} \leq C\left\{\left(f_{D_{1}}\left|u_{\varepsilon}\right|^{2}\right)^{1 / 2}+\sum_{|\alpha| \leq m-1}\left(f_{D_{1}}\left|f^{\alpha}\right|^{q}\right)^{1 / q}+\|G\|_{C^{m, \sigma}\left(D_{1}\right)}\right\},
$$

where $C$ depends only on $d, n, m, \mu, q, \sigma, \theta$ and $M_{1}$.

Similar to (1.6), estimate (1.11) is the $C^{m-1,1}$ estimate uniform down to the scale $\varepsilon$ for the operator $\mathcal{L}_{\varepsilon}$, which separates the large-scale estimates due to the homogenization process from the small-scale estimates related to the smoothness of the coefficients. If in addition, $A$ is Hölder continuous, i.e., there exist $\Lambda_{0}>0, \tau_{0} \in(0,1)$ such that

$$
|A(x)-A(y)| \leq \Lambda_{0}|x-y|^{\tau_{0}} \quad \text { for any } x, y \in \mathbb{R}^{d},
$$

we can derive the full-scale boundary $C^{m-1,1}$ estimate

$$
\left\|\nabla^{m} u_{\varepsilon}\right\|_{L^{\infty}\left(D_{1 / 4}\right)} \leq C\left\{\left(f_{D_{1}}\left|u_{\varepsilon}\right|^{2}\right)^{1 / 2}+\sum_{|\alpha| \leq m-1}\left(f_{D_{1}}\left|f^{\alpha}\right|^{q}\right)^{1 / q}+\|G\|_{C^{m, \sigma}\left(D_{1}\right)}\right\} .
$$


This generalizes the boundary Lipschitz estimates in $[5,35]$ for second-order elliptic systems to higher-order elliptic systems.

Note that Theorem 1.3 does not require the symmetry assumption on the coefficient matrix $A$. Therefore, it may be of some independent interests even for second-order elliptic systems $[21$, p. 485]. Recall that the symmetry assumption on the coefficient matrix $A$ is made in [23] to establish the uniform boundary Lipschitz estimate for second-order elliptic systems with Neumann boundary conditions. Such an assumption was removed in [3], where the boundary Lipschitz estimate was obtained for second-order elliptic systems with almost periodic coefficients. However, our investigations do not rely on the nontangential maximum function estimates, which had played an essential role in [3, p. 1896]. This may allow one to treat more general elliptic systems which arise in the homogenization theory [9], see also [21] for some discussions on this topic.

Finally, we mention that the requirements on smoothness of coefficients and the domain for uniform estimates in Theorems 1.1-1.3 are the same as those for second-order elliptic systems [35]. Therefore, results in theorems above, combined with the interior estimates in our previous paper [28], present a unified description on the uniform regularity estimates in homogenization of $2 m$-order elliptic systems in the divergence form. The counterpart for higher-order elliptic operators of non-divergence form will be studied in future.

The proofs of theorems above rely on the following convergence result.

Theorem 1.4 Suppose that $\Omega$ is a bounded Lipschitz domain in $\mathbb{R}^{d}, d \geq 1$, and the coefficient matrix A satisfies (1.2)-(1.3). Let $u_{\varepsilon}, u_{0}$ be the weak solutions to the Dirichlet problem (1.1) and the homogenized problem (2.2), respectively. Then, for $0<\varepsilon<1$ and any $0<v<1$, we have

$$
\left\|u_{\varepsilon}-u_{0}\right\|_{H_{0}^{m-1}(\Omega)} \leq C_{\nu} \varepsilon^{1-v}\left\{\|\dot{g}\|_{W A^{m, 2}(\partial \Omega)}+\|f\|_{H^{-m+1}(\Omega)}\right\},
$$

where $C_{v}$ depends only on $d, n, m, v, \mu$ and $\Omega$. If in addition $A$ is symmetric, i.e., $A=A^{*}$, then

$$
\left\|u_{\varepsilon}-u_{0}\right\|_{H_{0}^{m-1}(\Omega)} \leq C \varepsilon \ln (1 / \varepsilon)\left\{\|\dot{g}\|_{W A^{m, 2}(\partial \Omega)}+\|f\|_{H^{-m+1}(\Omega)}\right\},
$$

where $C$ depends only on $d, n, m, \mu$ and $\Omega$.

The error estimates above can be viewed as a counterpart in general Lipschitz domains for the convergence rates obtained in $[25,29,30,39]$. Estimate (1.14) is new even for secondorder elliptic systems. Recall that sharp convergence rate is also one of the central issues in quantitative homogenization theory. The estimate

$$
\left\|u_{\varepsilon}-u_{0}\right\|_{L^{2}(\Omega)} \leq C \varepsilon\left\|u_{0}\right\|_{H^{2}(\Omega)}
$$

has been obtained for second-order elliptic equations in divergence form in $C^{1,1}$ domains $[18,37,38]$, as well as in Lipschitz domains with additional assumptions $u_{0} \in H^{2}(\Omega)$ and $A=$ $A^{*}[28,35]$. In $[22,42]$, the $O[\varepsilon \ln (1 / \varepsilon)]$ convergence rate like (1.15) was obtained for secondorder elliptic systems under the assumption that $A=A^{*}$. Compared with the reference aforementioned, our estimate (1.14), although suboptimal, holds in general Lipschitz domains and needs neither the symmetry of $A$, nor additional regularity assumptions of $u_{0}$. Moreover, the assumptions on the regularity of $A, \dot{g}$ and $f$ are also rather general. To the best of the authors' knowledge, optimal or suboptimal convergence rate under such weak conditions seems to be unknown previously even for second-order elliptic systems. 
The proof of Theorem 1.4 follows the line of $[22,37]$. The first step is to derive an estimate like

$$
\left\|u_{\varepsilon}-u_{0}\right\|_{H^{m-1}(\Omega)} \leq C \varepsilon^{(1 / 2)^{-}}\left\{\|\dot{g}\|_{W A^{m, 2}(\partial \Omega)}+\|f\|_{H^{-m+1}(\Omega)}\right\} .
$$

When $A$ is symmetric, this was done with the help of the nontangential maximum function estimate, which gives proper controls on $u_{0}$ near the boundary $\partial \Omega$, see $[22,35,42]$ for the details. Unfortunately, if $A$ is not symmetric and the domain is just Lipschitz (or even $C^{1}$ ) the nontangential maximum function estimate is not in hand. Instead, we will take advantage of some weighted estimate of $u_{0}$ (see Lemma 3.2) to achieve the goal. With the estimate (1.16) at our disposal, we then modify the duality argument in [37] with proper weight to derive the desired convergence rate. This idea is also partially motivated by [22,42], see Remark 3.1.

Armed with Theorem 1.4, our proof of Theorems 1.1 and 1.3 follows the scheme in $[3,4,35]$, which roughly speaking is a three-step argument:

(i) Establish the convergence rate in $L^{2}(\Omega)$ in terms of boundary data $g$ and the forcing term $f$, i.e., the error estimate like

$$
\left\|u_{\varepsilon}-u_{0}\right\|_{L^{2}(\Omega)} \leq C \varepsilon^{\sigma_{0}}\{\text { norms of data } g \text { and } f\} \text { for some } 0<\sigma_{0} \leq 1 ;
$$

(ii) Prove that $u_{\varepsilon}$ satisfies the so-called flatness property, i.e., how well it could be approximated by affine functions as $u_{0}$ does;

(iii) Iterate step (ii) down to the scale $\varepsilon$, with the help of the error estimate in the first step.

Note that (1.14) gives (i), and we can thus pass to Step (ii). We shall adapt some ideas in $[3,4,35]$ to verify that $u_{\varepsilon}$ satisfies the "flatness property." However, instead of estimating how well $u_{\varepsilon}$ is approximated by affine functions as in $[3,4,35]$, we estimate how well $u_{\varepsilon}$ is approximated by polynomials of degree $m-1$ and $m$, respectively. By a proper iteration argument, we then derive the desired large-scale $C^{m-1, \lambda}(0<\lambda<1)$ and $C^{m-1,1}$ estimates. The corresponding full-scale estimates (4.14) and (6.16) follow from a standard blow-up argument.

Finally, the proof of Theorem 1.2 relies on the boundary Hölder estimate (1.6) and a real-variable argument originated from [11] and further developed in [31,32]. The key idea is to reduce the $W^{m, p}$ estimate (1.9) to a reverse Hölder inequality of the corresponding homogeneous problem, see Lemma 5.1 for the details.

\section{Preliminaries}

\subsection{Function spaces}

To begin with, let us give the definitions of some function spaces involved next. Let $\Omega$ be a bounded Lipschitz domain in $\mathbb{R}^{d}$. Let $H^{m}\left(\Omega ; \mathbb{R}^{n}\right)$ and $H_{0}^{m}\left(\Omega ; \mathbb{R}^{n}\right)$ with dual $H^{-m}\left(\Omega ; \mathbb{R}^{n}\right)$ be the conventional Sobolev spaces of $\mathbb{R}^{n}$-valued functions. For $0<s<1,1<p<\infty$ and any nonnegative integer $k$, let $B_{p}^{k+s}(\Omega)$ be the Besov space with norm (see e.g., [19, p. 17])

$$
\|u\|_{B_{p}^{k+s}(\Omega)}=\sum_{0 \leq \ell \leq k}\left\|\nabla^{\ell} u\right\|_{L^{p}(\Omega)}+\sum_{|\zeta|=k}\left\{\int_{\Omega} \int_{\Omega} \frac{\left|D^{\zeta} f(x)-D^{\zeta} f(y)\right|^{p}}{|x-y|^{d+s p}} \mathrm{~d} x \mathrm{~d} y\right\}^{1 / p} .
$$

Since $\Omega$ is a bounded Lipschitz domain, $B_{p}^{k+s}(\Omega)$ consists of the restrictions to $\Omega$ of functions in $B_{p}^{k+s}\left(\mathbb{R}^{d}\right)[19$, p. 25]. 
Also, define the Whitney-Besov space $\dot{B}_{p}^{m-1+s}\left(\partial \Omega ; \mathbb{R}^{n}\right)$ as the closure of the set of arrays

$$
\left\{\left\{D^{\alpha} \mathcal{U}\right\}_{|\alpha| \leq m-1}: \mathcal{U} \in C_{c}^{\infty}\left(\mathbb{R}^{d}\right)\right\}
$$

under the norm

$$
\|\dot{u}\|_{\dot{B}_{p}^{m-1+s}(\partial \Omega)}=\sum_{|\alpha| \leq m-1}\left\{\left\|u_{\alpha}\right\|_{L^{p}(\partial \Omega)}+\left(\int_{\partial \Omega} \int_{\partial \Omega} \frac{\left|u_{\alpha}(x)-u_{\alpha}(y)\right|^{p}}{|x-y|^{d-1+s p}} \mathrm{~d} S_{x} \mathrm{~d} S_{y}\right)^{1 / p}\right\},
$$

where $\dot{u}=\left\{u_{\alpha}\right\}_{|\alpha| \leq m-1}$, see e.g., [1].

Define the Whitney-Sobolev space $W A^{m, p}\left(\partial \Omega, \mathbb{R}^{n}\right)$ as the completion of the set of arrays of $\mathbb{R}^{n}$-valued functions

$$
\left\{\left\{\left.D^{\alpha} \mathcal{U}\right|_{\partial \Omega}\right\}_{|\alpha| \leq m-1}: \mathcal{U} \in C_{c}^{\infty}\left(\mathbb{R}^{d} ; \mathbb{R}^{n}\right)\right\},
$$

under the norm [26]

$$
\|\dot{g}\|_{W A^{m, p}(\partial \Omega)}=\sum_{|\alpha| \leq m-1}\left\|g_{\alpha}\right\|_{L^{p}(\partial \Omega)}+\sum_{|\alpha|=m-1}\left\|\nabla_{t a n} g_{\alpha}\right\|_{L^{p}(\partial \Omega)} .
$$

\subsection{Qualitative homogenization}

Under the ellipticity condition (1.2), for any $\dot{g} \in W A^{m, 2}\left(\partial \Omega, \mathbb{R}^{n}\right)$ and $f \in H^{-m}\left(\Omega ; \mathbb{R}^{n}\right)$, Dirichlet problem (1.1) admits a unique weak solution $u_{\varepsilon}$ in $H^{m}\left(\Omega ; \mathbb{R}^{n}\right)$ such that

$$
\left\|u_{\varepsilon}\right\|_{H^{m}(\Omega)} \leq C\left\{\|f\|_{H^{-m}(\Omega)}+\|\dot{g}\|_{W A^{m, 2}(\partial \Omega)}\right\},
$$

where $C$ depends only on $d, m, n, \mu$ and $\Omega$. It is known that (see e.g., [9,29]) under the additional periodicity condition (1.3), the operator $\mathcal{L}_{\varepsilon}$ is G-convergent to $\mathcal{L}_{0}$, where

$$
\left(\mathcal{L}_{0} u\right)_{i}=\sum_{|\alpha|=|\beta|=m}(-1)^{m} D^{\alpha}\left(\bar{A}_{i j}^{\alpha \beta} D^{\beta} u_{j}\right)
$$

is an elliptic operator of order $2 m$ with constant coefficients,

$$
\bar{A}_{i j}^{\alpha \beta}=\sum_{|\gamma|=m} \frac{1}{|Q|} \int_{Q}\left[A_{i j}^{\alpha \beta}(y)+A_{i \ell}^{\alpha \gamma}(y) D^{\gamma} \chi_{\ell j}^{\beta}(y)\right] \mathrm{d} y .
$$

Here, $Q=[0,1)^{d}, \chi=\left(\chi_{j}^{\gamma}\right)=\left(\chi_{i j}^{\gamma}\right)$ is the matrix of correctors for the operator $\mathcal{L}_{\varepsilon}$ given by

$$
\left\{\begin{array}{l}
\sum_{|\alpha|=|\beta|=m} D^{\alpha}\left\{A_{i k}^{\alpha \beta}(y) D^{\beta} \chi_{k j}^{\gamma}(y)\right\}=-\sum_{|\alpha|=m} D^{\alpha} A_{i j}^{\alpha \gamma}(y) \text { in } \mathbb{R}^{d}, \\
\chi_{j}^{\gamma}(y) \text { is 1-periodic and } \int_{Q} \chi_{j}^{\gamma}(y)=0 .
\end{array}\right.
$$

The matrix $\left(\bar{A}_{i j}^{\alpha \beta}\right)$ is bounded and satisfies the coercivity condition (1.2). Thus, the following homogenized problem of (1.1),

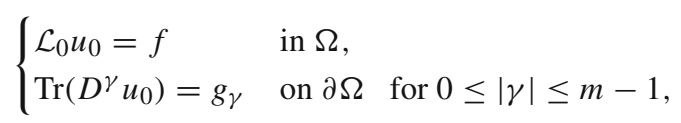

admits a unique weak solution $u_{0} \in H^{m}\left(\Omega ; \mathbb{R}^{n}\right)$, satisfying

$$
\left\|u_{0}\right\|_{H^{m}(\Omega)} \leq C\left\{\|f\|_{H^{-m}(\Omega)}+\|\dot{g}\|_{W A^{m, 2}(\partial \Omega)}\right\} .
$$


For $1 \leq i, j \leq n$ and multi-indexes $\alpha, \beta$ with $|\alpha|=|\beta|=m$, set

$$
B_{i j}^{\alpha \beta}(y)=A_{i j}^{\alpha \beta}(y)+\sum_{|\gamma|=m} A_{i k}^{\alpha \gamma}(y) D^{\gamma} \chi_{k j}^{\beta}(y)-\bar{A}_{i j}^{\alpha \beta} .
$$

By the definitions of $\chi^{\gamma}(y)$ and $\bar{A}$, for any $1 \leq i, j \leq n$ and any multi-indexes $\alpha, \beta$ with $|\alpha|=|\beta|=m, B_{i j}^{\alpha \beta}(y) \in L^{2}(Q)$ is 1-periodic with zero mean, and $\sum_{|\alpha|=m} D^{\alpha} B_{i j}^{\alpha \beta}(y)=0$. Therefore, there exists a function $\mathfrak{B}_{i j}^{\gamma \alpha \beta}$ such that

$$
\mathfrak{B}_{i j}^{\gamma \alpha \beta}=-\mathfrak{B}_{i j}^{\alpha \gamma \beta}, \quad \sum_{|\gamma|=m} D^{\gamma} \mathfrak{B}_{i j}^{\gamma \alpha \beta}=B_{i j}^{\alpha \beta} \quad \text { and }\left\|\mathfrak{B}_{i j}^{\gamma \alpha \beta}\right\|_{H^{m}(Q)} \leq C\left\|B_{i j}^{\alpha \beta}\right\|_{L^{2}(Q)},
$$

where $C$ depends only on $d, n, m$, see [28, Lemma 2.1].

Let $\mathcal{L}_{\varepsilon}^{*}$ be the adjoint operators of $\mathcal{L}_{\varepsilon}$, i.e.,

$$
\mathcal{L}_{\varepsilon}^{*}=(-1)^{m} \sum_{|\alpha|=|\beta|=m} D^{\alpha}\left(A^{* \alpha \beta}(x / \varepsilon) D^{\beta}\right), \quad A^{*}=\left(A_{i j}^{* \alpha \beta}\right)=\left(A_{j i}^{\beta \alpha}\right) .
$$

Parallel to (2.1), we introduce the matrix of correctors $\chi^{*}=\left(\chi_{j}^{* \gamma}\right)=\left(\chi_{i j}^{* \gamma}\right)$ for $\mathcal{L}_{\varepsilon}^{*}$,

$$
\left\{\begin{array}{l}
\sum_{|\alpha|=|\beta|=m} D^{\alpha}\left\{A_{i k}^{* \alpha \beta}(y) D^{\beta} \chi_{k j}^{* \gamma}(y)\right\}=-\sum_{|\alpha|=m} D^{\alpha} A_{i j}^{* \alpha \gamma}(y) \text { in } \mathbb{R}^{d}, \\
\chi_{j}^{* \gamma}(y) \text { is 1-periodic and } \int_{Q} \chi_{j}^{* \gamma}(y)=0 .
\end{array}\right.
$$

We also introduce the dual correctors $\mathfrak{B}^{* \gamma \alpha \beta}(y)$ of $\mathcal{L}_{\varepsilon}^{*}$. It is not difficult to see that $\chi^{* \gamma}$ and $\mathfrak{B}^{* \gamma \alpha \beta}$ satisfy the same properties as $\chi^{\gamma}$ and $\mathfrak{B}^{\gamma \alpha \beta}$, since $A^{*}$ satisfies the same conditions as $A$.

\subsection{Smoothing operators and auxiliary estimates}

For any fixed $\varphi \in C_{c}^{\infty}\left(B\left(0, \frac{1}{2}\right)\right)$ such that $\varphi>0$ and $\int_{\mathbb{R}^{d}} \varphi(x) \mathrm{d} x=1$, set $\varphi_{\varepsilon}=\frac{1}{\varepsilon^{d}} \varphi\left(\frac{x}{\varepsilon}\right)$ and define

$$
S_{\varepsilon}(f)(x)=\int_{\mathbb{R}^{d}} \varphi_{\varepsilon}(x-y) f(y) \mathrm{d} y, \quad \text { and } \quad S_{\varepsilon}^{2}=S_{\varepsilon} \circ S_{\varepsilon} .
$$

Denote $\delta(x)=\operatorname{dist}(x, \partial \Omega), \Omega^{\varepsilon}=\{x \in \Omega: \delta(x)>\varepsilon\}$ and $\Omega_{\varepsilon}=\{x \in \Omega: \delta(x)<\varepsilon\}$.

Lemma 2.1 Assume that $f \in L^{p}\left(\mathbb{R}^{d}\right)$ for some $1 \leq p<\infty$ and $g \in L_{\text {loc }}^{p}\left(\mathbb{R}^{d}\right)$ is 1-periodic. Let $h \in L^{\infty}\left(\mathbb{R}^{d}\right)$ with compact support $\Omega^{3 \varepsilon}$. Then,

$$
\begin{aligned}
& \left\|g(x / \varepsilon) S_{\varepsilon}(f)(x) h(x)\right\|_{L^{p}\left(\Omega^{3 \varepsilon} ; \delta\right)} \leq C\|h\|_{L^{\infty}}\|g\|_{L^{p}(Q)}\|f\|_{L^{p}\left(\Omega^{2 \varepsilon} ; \delta\right)}, \\
& \left\|g(x / \varepsilon) S_{\varepsilon}(f)(x) h(x)\right\|_{L^{p}\left(\Omega^{3 \varepsilon} ; \delta^{-1}\right)} \leq C\|h\|_{L^{\infty}}\|g\|_{L^{p}(Q)}\|f\|_{L^{p}\left(\Omega^{2 \varepsilon} ; \delta^{-1}\right)},
\end{aligned}
$$

where $\|u\|_{L^{p}(\Omega ; \delta)}$ (similar for $\left.\|u\|_{L^{p}\left(\Omega ; \delta^{-1}\right)}\right)$ denotes the weighted norm

$$
\|u\|_{L^{p}(\Omega ; \delta)}=\left(\int_{\Omega}|u(x)|^{p} \delta(x) d x\right)^{1 / p} .
$$


Proof Observe that

$$
\begin{aligned}
\int_{\mathbb{R}^{d}}\left|g(x / \varepsilon) h(x) \int_{\mathbb{R}^{d}} \varphi_{\varepsilon}(x-y) f(y) \mathrm{d} y\right|^{p} \delta(x) \mathrm{d} x \\
\leq C \int_{\Omega^{3 \varepsilon}}|g(x / \varepsilon)|^{p} \int_{\Omega^{2 \varepsilon}} \varphi_{\varepsilon}(x-y)|f(y)|^{p} \delta(y) \\
\quad \times \mathrm{d} y\left\{\int_{\Omega^{2 \varepsilon}} \varphi_{\varepsilon}(x-y) \delta(y)^{-q / p} \mathrm{~d} y\right\}^{p / q} \delta(x) \mathrm{d} x \\
\leq C \int_{\Omega^{2 \varepsilon}} \int_{\Omega^{3 \varepsilon}}|g(x / \varepsilon)|^{p} \varphi_{\varepsilon}(x-y) \mathrm{d} x|f(y)|^{p} \delta(y) \mathrm{d} y \\
\leq C \int_{Q}|g(z)|^{p} d z \int_{\Omega^{2 \varepsilon}}|f(y)|^{p} \delta(y) \mathrm{d} y,
\end{aligned}
$$

where we have used Fubini's theorem and the observation

$$
\int_{\Omega^{2 \varepsilon}} \varphi_{\varepsilon}(x-y)[\delta(y)]^{-q / p} \mathrm{~d} y \leq C[\delta(x)]^{-q / p}
$$

for the second inequality. This gives (2.6). The proof of (2.7) is the same.

Lemma 2.2 Let $\widetilde{\Omega}_{\varepsilon}=\left\{x \in \mathbb{R}^{d}: \delta(x)<\varepsilon\right\}, f \in H^{\ell}\left(\mathbb{R}^{d}\right), \ell \geq 0$. Then, for any multi-index $\alpha,|\alpha|=\ell$,

$$
\begin{aligned}
& \left\|S_{\varepsilon}\left(D^{\alpha} f\right)\right\|_{L^{p}\left(\Omega_{\varepsilon}\right)} \leq C \varepsilon^{-\ell}\|f\|_{L^{p}\left(\widetilde{\Omega}_{2 \varepsilon}\right)}, \\
& \left\|S_{\varepsilon}\left(D^{\alpha} f\right)\right\|_{L^{p}\left(\Omega^{3 \varepsilon} ; \delta\right)} \leq C \varepsilon^{-\ell}\|f\|_{L^{p}\left(\Omega^{\varepsilon} ; \delta\right)} .
\end{aligned}
$$

Proof Inequality (2.9) was proved in [28, Lemma 2.3], and the proof of (2.10) is quite similar. We provide it just for completeness.

$$
\begin{aligned}
\left\|S_{\varepsilon}\left(D^{\alpha} f\right)\right\|_{L^{p}\left(\Omega^{3 \varepsilon} ; \delta\right)}^{p}=\int_{\Omega^{3 \varepsilon}}\left|\int_{\mathbb{R}^{d}} D^{\alpha} \varphi_{\varepsilon}(x-y) f(y) \mathrm{d} y\right|^{p} \delta(x) \mathrm{d} x \\
\leq \int_{\Omega^{3 \varepsilon}} \int_{\Omega^{2 \varepsilon}}\left|D^{\alpha} \varphi_{\varepsilon}(x-y)\right||f(y)|^{p} \delta(y) \\
\quad \times \mathrm{d} y\left\{\int_{\Omega^{2 \varepsilon}}\left|D^{\alpha} \varphi_{\varepsilon}(x-y)\right|[\delta(y)]^{-q / p} \mathrm{~d} y\right\}^{p / q} \delta(x) \mathrm{d} x \\
\leq \frac{C}{\varepsilon^{p \ell}} \int_{\Omega^{2 \varepsilon}}|f(y)|^{p} \delta(y) \mathrm{d} y,
\end{aligned}
$$

where, for the last step, we have used Fubini's theorem and the observation

$$
\begin{aligned}
\int_{\Omega^{2 \varepsilon}}\left|D^{\alpha} \varphi_{\varepsilon}(x-y)\right|[\delta(y)]^{-q / p} \mathrm{~d} y & \leq C \int_{\Omega^{2 \varepsilon}}\left|D^{\alpha} \varphi_{\varepsilon}(x-y)\right|[\delta(x)]^{-q / p} \mathrm{~d} y \\
& \leq C \varepsilon^{-\ell}[\delta(x)]^{-q / p}
\end{aligned}
$$

for $x \in \Omega^{3 \varepsilon}$.

Lemma 2.3 Suppose that $f \in W^{1, q}\left(\mathbb{R}^{d}\right)$ for some $1<q<\infty$. Let $\nabla^{s} f=\left(D^{\alpha} f\right)_{|\alpha|=s}$. Then,

$$
\left\|S_{\varepsilon}(f)-f\right\|_{L^{q}\left(\Omega^{2 \varepsilon} ; \delta\right)} \leq C \varepsilon\|\nabla f\|_{L^{q}\left(\Omega^{\varepsilon} ; \delta\right)} .
$$

Proof See [42, Lemma 3.3] and also [35, Lemma 2.2] for the case $q=2$. 
Lemma 2.4 Assume that A satisfies (1.2)-(1.3), and $u_{\varepsilon} \in H^{m}\left(B\left(x_{0}, R\right) \cap \Omega ; \mathbb{R}^{n}\right)$ is a solution to $\mathcal{L}_{\varepsilon} u=\sum_{|\alpha| \leq m} D^{\alpha} f^{\alpha}$ in $B\left(x_{0}, R\right) \cap \Omega$ with $\operatorname{Tr}\left(D^{\gamma} u_{\varepsilon}\right)=D^{\gamma} G$ on $B\left(x_{0}, R\right) \cap \partial \Omega$ for some $G \in H^{m}\left(B\left(x_{0}, R\right) \cap \Omega ; \mathbb{R}^{n}\right)$ where $x_{0} \in \partial \Omega$. Let $f^{\alpha} \in L^{2}\left(B\left(x_{0}, R\right) \cap \Omega ; \mathbb{R}^{n}\right)$ for $|\alpha| \leq m$. Then, for $0 \leq j \leq m$ and $0<r<R$, we have

$$
\begin{aligned}
& \int_{B\left(x_{0}, r\right) \cap \Omega}\left|\nabla^{j}\left(u_{\varepsilon}-G\right)\right|^{2} \\
& \leq \frac{C}{(R-r)^{2 j}} \int_{B\left(x_{0}, R\right) \cap \Omega}\left(\left|u_{\varepsilon}\right|^{2}+|G|^{2}\right)+C R^{2 m-2 j} \int_{B\left(x_{0}, R\right) \cap \Omega}\left|\nabla^{m} G\right|^{2} \\
& \quad+C \sum_{|\alpha| \leq m} R^{4 m-2 j-2|\alpha|} \int_{B\left(x_{0}, R\right) \cap \Omega}\left|f^{\alpha}\right|^{2},
\end{aligned}
$$

where $C$ depends only on $d, n, m, \mu$ and $\Omega$.

Proof It is obvious that $v_{\varepsilon}=u_{\varepsilon}-G$ is a solution to

$$
\begin{aligned}
& \mathcal{L}_{\varepsilon} v_{\varepsilon}=\sum_{|\alpha| \leq m} D^{\alpha} f^{\alpha}+\sum_{|\alpha|=|\beta|=m} D^{\alpha}\left\{A^{\alpha \beta}(x / \varepsilon) D^{\beta} G\right\} \quad \text { in } B\left(x_{0}, R\right) \cap \Omega, \\
& \operatorname{Tr}\left(D^{\gamma} v_{\varepsilon}\right)=0 \quad \text { on } B\left(x_{0}, R\right) \cap \partial \Omega \quad \text { for } 0 \leq|\gamma| \leq m-1 .
\end{aligned}
$$

Let $\phi \in C_{c}^{\infty}\left(B\left(x_{0}, R\right)\right)$ with $\phi=1$ in $B\left(x_{0}, r\right)$ and $\left|\nabla^{k} \phi\right| \leq C(R-r)^{-k}$. Multiplying $v_{\varepsilon} \phi^{2}$ and using integration by parts, we obtain that

$$
\begin{aligned}
\int_{B\left(x_{0}, R\right) \cap \Omega}\left|\nabla^{m} v_{\varepsilon}\right|^{2} \phi^{2} \leq & \sum_{|\alpha| \leq m}\left\{C\left(\epsilon_{0}\right) R^{2 m-2|\alpha|} \int_{B\left(x_{0}, R\right) \cap \Omega}\left|f^{\alpha}\right|^{2}\right. \\
& \left.+\frac{\epsilon_{0}}{R^{2 m-2|\alpha|}} \int_{B\left(x_{0}, R\right) \cap \Omega}\left|D^{\alpha}\left(v_{\varepsilon} \phi^{2}\right)\right|^{2}\right\} \\
& +C\left(\epsilon_{0}\right) \sum_{|\alpha|=m} \int_{B\left(x_{0}, R\right) \cap \Omega}\left|D^{\alpha} G\right|^{2}+\epsilon_{0} \int_{B\left(x_{0}, R\right) \cap \Omega}\left|\nabla^{m} v_{\varepsilon}\right|^{2} \phi^{2} \\
& +\sum_{j=0}^{m-1} \frac{C \epsilon_{0}+C}{(R-r)^{2 m-2 j}} \int_{\left(B\left(x_{0}, R\right) \backslash B\left(x_{0}, r\right)\right) \cap \Omega}\left|\nabla^{j} v_{\varepsilon}\right|^{2} .
\end{aligned}
$$

Note that $v_{\varepsilon} \phi^{2} \in H_{0}^{m}\left(B\left(x_{0}, R\right) \cap \Omega\right)$. Using Poincaré's inequality and setting $\epsilon_{0}$ small enough, we may obtain from (2.13) that

$$
\begin{aligned}
\int_{B\left(x_{0}, r\right) \cap \Omega}\left|\nabla^{m}\left(u_{\varepsilon}-G\right)\right|^{2} \leq & \sum_{j=0}^{m-1} \frac{C}{(R-r)^{2 m-2 j}} \int_{\left(B\left(x_{0}, R\right) \backslash B\left(x_{0}, r\right)\right) \cap \Omega}\left|\nabla^{j}\left(u_{\varepsilon}-G\right)\right|^{2} \\
& +C\left\{\sum_{|\alpha| \leq m} R^{2 m-2|\alpha|} \int_{B\left(x_{0}, R\right) \cap \Omega}\left|f^{\alpha}\right|^{2}\right. \\
& \left.+\sum_{|\alpha|=m} \int_{B\left(x_{0}, R\right) \cap \Omega}\left|D^{\alpha} G\right|^{2}\right\}
\end{aligned}
$$

where $C$ depends only on $d, n, m$ and $\mu$, but never on $\varepsilon, R$. The estimate (2.12) follows from (2.14) in the same way as Corollary 23 in [8] through an induction argument. 
Remark 2.1 It is possible to replace the $L^{2}$ norm of $f^{\alpha}$ in (2.12) by the $L^{p}$ norm for some $1<p<2$ when $|\alpha|<m$. For example, assuming that $f^{\alpha}=0$ for $1 \leq|\alpha| \leq m$, we may prove that

$$
\begin{aligned}
\int_{B\left(x_{0}, r\right) \cap \Omega}\left|\nabla^{j}\left(u_{\varepsilon}-G\right)\right|^{2} \leq & \frac{C}{(R-r)^{2 j}} \int_{B\left(x_{0}, R\right) \cap \Omega}\left(\left|u_{\varepsilon}\right|^{2}+|G|^{2}\right) \\
& +C R^{2 m-2 j} \int_{B\left(x_{0}, R\right) \cap \Omega}\left|\nabla^{m} G\right|^{2} \\
& +C R^{4 m-2 j+d-\frac{2 d}{p}}\left(\int_{B\left(x_{0}, R\right) \cap \Omega}\left|f^{0}\right|^{p}\right)^{\frac{2}{p}} \\
& \text { for } p>\max \{1,2 d /(d+2 m)\} .
\end{aligned}
$$

\section{Convergence rates in Lipschitz domains}

Let $0 \leq \rho_{\varepsilon} \leq 1$ be a function in $C_{c}^{\infty}(\Omega)$ with $\operatorname{supp}\left(\rho_{\varepsilon}\right) \subset \Omega^{3 \varepsilon}, \rho_{\varepsilon}=1$ on $\Omega^{4 \varepsilon}$ and $\left|\nabla^{m} \rho_{\varepsilon}\right| \leq C \varepsilon^{-m}$.

Lemma 3.1 Suppose that $\Omega$ is a bounded Lipschitz domain in $\mathbb{R}^{d}$, and A satisfies (1.2)-(1.3). Let $u_{\varepsilon}, u_{0}$ be the weak solutions to Dirichlet problems (1.1) and (2.2), respectively. Define

$$
w_{\varepsilon}=u_{\varepsilon}-u_{0}-\varepsilon^{m} \sum_{|\gamma|=m} \chi^{\gamma}(x / \varepsilon) S_{\varepsilon}^{2}\left(D^{\gamma} u_{0}\right) \rho_{\varepsilon} .
$$

Then, for any $\phi \in H_{0}^{m}\left(\Omega ; \mathbb{R}^{n}\right)$, we have

$$
\begin{aligned}
& \left|\sum_{|\alpha|=|\beta|=m} \int_{\Omega} D^{\alpha} \phi_{i} A_{i j}^{\alpha \beta}(x / \varepsilon) D^{\beta} w_{\varepsilon j}\right| \\
& \leq C\left\|\nabla^{m} \phi\right\|_{L^{2}\left(\Omega_{4 \varepsilon}\right)}\left\|\nabla^{m} u_{0}\right\|_{L^{2}\left(\Omega_{4 \varepsilon}\right)} \\
& \quad+C\left\|\nabla^{m} \phi\right\|_{L^{2}\left(\Omega_{4 \varepsilon}\right)} \sum_{0 \leq k \leq m-1,} \varepsilon^{k}\left\|S_{\varepsilon}\left(\nabla^{m+k} u_{0}\right)\right\|_{L^{2}\left(\Omega_{5 \varepsilon} \backslash \Omega_{2 \varepsilon}\right)} \\
& \quad+C\left\|\nabla^{m} \phi\right\|_{L^{2}\left(\Omega^{2 \varepsilon} ; \vartheta-1\right)}\left\|\nabla^{m} u_{0}-S_{\varepsilon}\left(\nabla^{m} u_{0}\right)\right\|_{L^{2}\left(\Omega^{2 \varepsilon} ; \vartheta\right)} \\
& \quad+C\left\|\nabla^{m} \phi\right\|_{L^{2}\left(\Omega^{2 \varepsilon} ; \vartheta-1\right)} \sum_{0 \leq k \leq m-1} \varepsilon^{m-k}\left\|S_{\varepsilon}\left(\nabla^{2 m-k} u_{0}\right)\right\|_{L^{2}\left(\Omega^{2 \varepsilon} ; \vartheta\right)},
\end{aligned}
$$

where $\vartheta(x)=\delta(x)$ or $1, C$ depends only on $d, n, m, \mu$ and $\Omega$.

Proof See [28, Lemma 3.1] for $\vartheta \equiv 1$. The proof for the case $\vartheta(x)=\delta(x)$ is almost the same with the help of Lemmas 2.1, 2.2 and 2.3.

Lemma 3.2 Let $\Omega$ be a bounded Lipschitz domain in $\mathbb{R}^{d}$. Let A satisfy (1.2)-(1.3) and let $u_{0}$ be the weak solution to homogenized problem (2.2) with $\dot{g} \in W A^{m, 2}\left(\partial \Omega ; \mathbb{R}^{n}\right), f \in$ $H^{-m+1}\left(\Omega ; \mathbb{R}^{n}\right)$. Then, for any $0<v<1 / 2$,

$$
\begin{aligned}
& \left\|\nabla^{m} u_{0}\right\|_{L^{2}\left(\Omega_{2 \varepsilon}\right)} \leq C_{\nu} \varepsilon^{1 / 2-v}\left\{\|\dot{g}\|_{W A^{m, 2}(\partial \Omega)}+\|f\|_{H^{-m+1}(\Omega)}\right\}, \\
& \left\|\nabla^{m+1} u_{0}\right\|_{L^{2}\left(\Omega^{2 \varepsilon}\right)} \leq C_{\nu} \varepsilon^{-1 / 2-v}\left\{\|\dot{g}\|_{W A^{m, 2}(\partial \Omega)}+\|f\|_{H^{-m+1}(\Omega)}\right\}, \\
& \left\|\nabla^{m} u_{0}\right\|_{L^{2}\left(\Omega^{2 \varepsilon} ; \delta^{-1}\right)} \leq C_{\nu} \varepsilon^{-v}\left\{\|\dot{g}\|_{W A^{m, 2}(\partial \Omega)}+\|f\|_{H^{-m+1}(\Omega)}\right\}, \\
& \left\|\nabla^{m+1} u_{0}\right\|_{L^{2}\left(\Omega^{2 \varepsilon} ; \delta\right)} \leq C_{\nu} \varepsilon^{-v}\left\{\|\dot{g}\|_{W A^{m, 2}(\partial \Omega)}+\|f\|_{H^{-m+1}(\Omega)}\right\},
\end{aligned}
$$


where $C_{v}$ depends only on $d, n, m, v, \mu$ and $\Omega$.

Proof Recall that $f \in H^{-m+1}(\Omega)$ can be written as

$$
f=\sum_{|\zeta| \leq m-1} D^{\zeta} f^{\zeta} \text { with }\|f\|_{H^{-m+1}(\Omega)} \approx \sum_{|\zeta| \leq m-1}\left\|f^{\zeta}\right\|_{L^{2}(\Omega)} .
$$

Let $\widetilde{f^{\zeta}}$ be the extension of $f^{\zeta}$, being zero in $\mathbb{R}^{d} \backslash \Omega$. Let $\widehat{\Omega}$ be a smooth bounded domain such that $\Omega \subset \widehat{\Omega}$. Let $v_{0}$ be the solution to

$$
\mathcal{L}_{0} v_{0}=\sum_{|\zeta| \leq m-1} D^{\zeta} \widetilde{f}^{\zeta} \quad \text { in } \widehat{\Omega}, \quad \operatorname{Tr}\left(D^{\gamma} v_{0}\right)=0 \quad \text { on } \partial \widehat{\Omega} \quad \text { for } 0 \leq|\gamma| \leq m-1 .
$$

Standard regularity estimates for higher-order elliptic systems imply that

$$
\sum_{1 \leq \ell \leq m+1}\left\|\nabla^{\ell} v_{0}\right\|_{L^{2}(\widehat{\Omega})} \leq C\|f\|_{H^{-m+1}(\Omega)} .
$$

Denote $\Sigma_{t}=\{x \in \Omega: \operatorname{dist}(x, \Omega)=t\}, 0 \leq t \leq c_{0}$. Similar to [28, Theorem 3.1] (see (3.23) and (3.24) therein), by trace theorem, we may prove that

$$
\begin{aligned}
& \sum_{1 \leq \ell \leq m}\left\|\nabla^{\ell} v_{0}\right\|_{L^{2}\left(\Sigma_{t}\right)} \leq C\|f\|_{H^{-m+1}(\Omega)}, \\
& \sum_{1 \leq \ell \leq m}\left\|\nabla^{\ell} v_{0}\right\|_{L^{2}\left(\Omega_{\varepsilon}\right)} \leq C \varepsilon^{1 / 2}\|f\|_{H^{-m+1}(\Omega)} .
\end{aligned}
$$

On the other hand, setting $u_{0}(x)=v_{0}(x)+v(x)$, we have

$$
\mathcal{L}_{0} v=0 \quad \text { in } \Omega, \quad \operatorname{Tr}\left(D^{\gamma} v\right)=g_{\gamma}-D^{\gamma} v_{0} \quad \text { on } \partial \Omega \quad \text { for } 0 \leq|\gamma| \leq m-1 .
$$

Thanks to Theorem $3^{\prime}$ and Theorem $5^{\prime}$ in [1], we have $v \in B_{2}^{m-1 / 2+s}(\Omega)$ for any $1 / 2<s<1$, and,

$$
\begin{aligned}
\|v\|_{B_{2}^{m-1 / 2+s}(\Omega)} & \leq C_{S}\left\{\|\dot{g}\|_{W A^{m, 2}(\partial \Omega)}+\left\|\dot{v}_{0}\right\|_{W A^{m, 2}(\partial \Omega)}\right\} \\
& \leq C_{S}\left\{\|\dot{g}\|_{W A^{m, 2}(\partial \Omega)}+\|f\|_{H^{-m+1}(\Omega)}\right\},
\end{aligned}
$$

where $\dot{v}_{0}=\left\{\left.D^{\gamma} v_{0}\right|_{\partial \Omega}\right\}_{|\gamma| \leq m-1}$, and (3.9) has been used for the last step. Therefore, we have $D^{\alpha} v \in B_{2}^{s-1 / 2}(\Omega)$ for $|\alpha|=m$. Thanks to Theorems 1.4.2.4 and 1.4.4.4 in [19],

$$
\int_{\Omega}\left|\nabla^{m} v(x)\right|^{2} \delta(x)^{1-2 s} \mathrm{~d} x \leq C_{s}\|v\|_{B_{2}^{m-1 / 2+s}(\Omega)}^{2} \leq C_{s}\left\{\|\dot{g}\|_{W A^{m, 2}(\partial \Omega)}^{2}+\|f\|_{H^{-m+1}(\Omega)}^{2}\right\} .
$$

This implies that

$$
\begin{aligned}
\int_{\Omega_{2 \varepsilon}}\left|\nabla^{m} v(x)\right|^{2} \mathrm{~d} x & =\int_{\Omega_{2 \varepsilon}}\left|\nabla^{m} v(x)\right|^{2} \delta(x)^{1-2 s} \delta(x)^{2 s-1} \mathrm{~d} x \\
& \leq C_{s} \varepsilon^{2 s-1}\left\{\|\dot{g}\|_{W A^{m, 2}(\partial \Omega)}^{2}+\|f\|_{H^{-m+1}(\Omega)}^{2}\right\}
\end{aligned}
$$

for any $1 / 2<s<1$. By combining (3.10) with (3.14), we derive (3.3) by setting $v=1-s$.

In view of (3.11) and interior estimates for higher-order elliptic systems with constant coefficients, we have

$$
\left|\nabla^{m+1} v(x)\right| \leq \frac{C}{\delta(x)}\left(f_{B\left(x, \frac{\delta(x)}{8}\right)}\left|\nabla^{m} v\right|^{2}\right)^{1 / 2} .
$$


Thus, by using (3.13) we deduce that

$$
\begin{aligned}
\left\|\nabla^{m+1} v\right\|_{L^{2}\left(\Omega^{2 \varepsilon}\right)}^{2} & \leq C \int_{\Omega^{2 \varepsilon}} \frac{1}{\delta(x)^{3-2 s}} f_{B\left(x, \frac{\delta(x)}{8}\right)}\left|\nabla^{m} v(y)\right|^{2} \delta(y)^{1-2 s} \mathrm{~d} y \mathrm{~d} x \\
& \leq C \varepsilon^{2 s-3}\left\|\nabla^{m} v\right\|_{L^{2}\left(\Omega ; \delta^{1-2 s}\right)}^{2} \\
& \leq C_{s} \varepsilon^{2 s-3}\left\{\|\dot{g}\|_{W A^{m, 2}(\partial \Omega)}^{2}+\|f\|_{H^{-m+1}(\Omega)}^{2}\right\},
\end{aligned}
$$

which, together with (3.8), gives (3.4).

For (3.5), it is easy to conclude from (3.12) and (3.13) that

$$
\begin{aligned}
\int_{\Omega^{2 \varepsilon}}\left|\nabla^{m} v(x)\right|^{2} \delta(x)^{-1} \mathrm{~d} x & =\int_{\Omega^{2 \varepsilon}}\left|\nabla^{m} v(x)\right|^{2} \delta(x)^{1-2 s} \delta(x)^{2 s-2} \mathrm{~d} x \\
& \leq C_{s} \varepsilon^{2 s-2}\left\{\|\dot{g}\|_{W A^{m, 2}(\partial \Omega)}^{2}+\|f\|_{H^{-m+1}(\Omega)}^{2}\right\} .
\end{aligned}
$$

On the other hand, by the co-area formula and (3.9) we deduce that

$$
\begin{aligned}
\int_{\Omega^{2 \varepsilon}}\left|\nabla^{m} v_{0}(x)\right|^{2} \delta(x)^{-1} \mathrm{~d} x & =\int_{\Omega^{2 \varepsilon} \backslash \Omega^{c_{0}}}\left|\nabla^{m} v_{0}(x)\right|^{2} \delta(x)^{-1} \mathrm{~d} x+\int_{\Omega^{c_{0}}}\left|\nabla^{m} v_{0}(x)\right|^{2} \delta(x)^{-1} \mathrm{~d} x \\
& \leq C \int_{2 \varepsilon}^{c_{0}} \int_{\Sigma_{t}}\left|\nabla^{m} v_{0}(x)\right|^{2} \frac{1}{t} \mathrm{~d} S \mathrm{~d} t+C \int_{\Omega^{c_{0}}}\left|\nabla^{m} v_{0}(x)\right|^{2} \mathrm{~d} x \\
& \leq C \ln (1 / \varepsilon)\left\{\|\dot{g}\|_{W A^{m, 2}(\partial \Omega)}^{2}+\|f\|_{H^{-m+1}(\Omega)}^{2}\right\}
\end{aligned}
$$

for $0<\varepsilon<1 / 2$. This, combined with (3.16), gives (3.5). The proof for (3.6) is almost the same as (3.4), and thus we omit the details.

Lemma 3.3 Suppose that the assumptions of Lemma 3.2 are satisfied, and $A$ is symmetric, i.e., $A=A^{*}$. Then, we have

$$
\begin{gathered}
\left\|\nabla^{m} u_{0}\right\|_{L^{2}\left(\Omega_{2 \varepsilon}\right)} \leq C \varepsilon^{1 / 2}\left\{\|\dot{g}\|_{W A^{m, 2}(\partial \Omega)}+\|f\|_{H^{-m+1}(\Omega)}\right\}, \\
\left\|\nabla^{m} u_{0}\right\|_{L^{2}\left(\Omega^{2 \varepsilon} ; \delta^{-1}\right)} \leq C[\ln (1 / \varepsilon)]^{1 / 2}\left\{\|\dot{g}\|_{W A^{m, 2}(\partial \Omega)}+\|f\|_{H^{-m+1}(\Omega)}\right\}, \\
\left\|\nabla^{m+1} u_{0}\right\|_{L^{2}\left(\Omega^{2 \varepsilon} ; \delta\right)} \leq C[\ln (1 / \varepsilon)]^{1 / 2}\left\{\|\dot{g}\|_{W A^{m, 2}(\partial \Omega)}+\|f\|_{H^{-m+1}(\Omega)}\right\},
\end{gathered}
$$

where $C$ depends only on $d, n, m, \mu$ and $\Omega$.

Proof The proof is the same as that of Lemma 3.2 except for three places. Firstly, since $A$ is symmetric, in place of (3.13) we have the nontangential maximum function estimate, see e.g., [41, Theorem 6.1],

$$
\left\|\mathcal{M}\left(\nabla^{m} v\right)\right\|_{L^{2}(\partial \Omega)} \leq C\|\dot{v}\|_{W A^{m, 2}(\partial \Omega)} \leq C\left\{\|\dot{g}\|_{W A^{m, 2}(\partial \Omega)}+\|f\|_{H^{-m+1}(\Omega)}\right\},
$$

where $\mathcal{M}\left(\nabla^{m} v\right)$ denotes the nontangential maximal function of $\nabla^{m} v$. Therefore, instead of (3.14) we have

$$
\int_{\Omega_{2 \varepsilon}}\left|\nabla^{m} v(x)\right|^{2} \mathrm{~d} x \leq C \varepsilon\left\{\|\dot{g}\|_{W A^{m, 2}(\partial \Omega)}^{2}+\|f\|_{H^{-m+1}(\Omega)}^{2}\right\},
$$

which, combined with (3.10), implies (3.18).

Secondly, in substitution for (3.16) we use the nontangential estimate (3.21) and the coarea formula to deduce that

$$
\begin{aligned}
\int_{\Omega^{2 \varepsilon}}\left|\nabla^{m} v(x)\right|^{2} \delta(x)^{-1} \mathrm{~d} x & \leq C \int_{2 \varepsilon}^{c_{0}} \int_{\Sigma_{t}} \frac{1}{t}\left|\nabla^{m} v(x)\right|^{2} d S d t+C \int_{\Omega^{c_{0}}}\left|\nabla^{m} v(x)\right|^{2} \mathrm{~d} x \\
& \leq C \ln (1 / \varepsilon)\left\{\|\dot{g}\|_{W A^{m, 2}(\partial \Omega)}^{2}+\|f\|_{H^{-m+1}(\Omega)}^{2}\right\},
\end{aligned}
$$


which, combined with (3.17), gives (3.19).

Finally, instead of (3.15), we have

$$
\begin{aligned}
\left\|\nabla^{m+1} v\right\|_{L^{2}\left(\Omega^{\varepsilon} ; \delta\right)}^{2} \leq & C \int_{\Omega^{\varepsilon} \backslash \Omega^{c_{0}}} \frac{1}{\delta(x)} f_{B\left(x, \frac{\delta(x)}{8}\right)}\left|\nabla^{m} v(y)\right|^{2} \mathrm{~d} y \mathrm{~d} x \\
& +C \int_{\Omega^{c_{0}}} f_{B\left(x, \frac{\delta(x)}{8}\right)}\left|\nabla^{m} v(y)\right|^{2} \mathrm{~d} y \mathrm{~d} x \\
\leq & C \int_{\varepsilon}^{c_{0}} \int_{\Sigma_{t}} \frac{1}{t}\left|\mathcal{M}\left(\nabla^{m} v\right)\right|^{2} \mathrm{~d} S \mathrm{~d} t+C\left\{\|\dot{g}\|_{W A^{m, 2}(\partial \Omega)}^{2}+\|f\|_{H^{-m+1}(\Omega)}^{2}\right\} \\
\leq & C \ln (1 / \varepsilon)\left\{\|\dot{g}\|_{W A^{m, 2}(\partial \Omega)}^{2}+\|f\|_{H^{-m+1}(\Omega)}^{2}\right\} .
\end{aligned}
$$

This, together with (3.8), gives (3.20). The proof is thus completed.

Lemma 3.4 Assume that $\Omega$ is a bounded Lipschitz domain in $\mathbb{R}^{d}$ and A satisfies (1.2)-(1.3). Let $u_{\varepsilon}, u_{0}$ be the weak solutions to Dirichlet problems (1.1) and (2.2), respectively, with $\dot{g} \in W A^{m, 2}\left(\partial \Omega ; \mathbb{R}^{n}\right), f \in H^{-m+1}\left(\Omega ; \mathbb{R}^{n}\right)$. Let $w_{\varepsilon}$ be defined as in (3.1). Then, for any $0<v<1 / 2$, we have

$$
\left\|w_{\varepsilon}\right\|_{H_{0}^{m}(\Omega)} \leq C_{\nu} \varepsilon^{1 / 2-v}\left\{\|\dot{g}\|_{W A^{m, 2}(\partial \Omega)}+\|f\|_{H^{-m+1}(\Omega)}\right\},
$$

where $C_{v}$ depends only on $d, n, m, v, \mu$ and $\Omega$. If in addition $A$ is symmetric, i.e., $A=A^{*}$, then

$$
\left\|w_{\varepsilon}\right\|_{H_{0}^{m}(\Omega)} \leq C \varepsilon^{1 / 2}\left\{\|\dot{g}\|_{W A^{m, 2}(\partial \Omega)}+\|f\|_{H^{-m+1}(\Omega)}\right\},
$$

where $C$ depends only on $d, n, m, \mu$ and $\Omega$.

Proof The estimate (3.23) has been proved in [28, Theorem 3.1], we only need to consider (3.22) here. Using Lemmas 2.2 and 2.3, we deduce from (3.2) that

$$
\begin{aligned}
& \left|\sum_{|\alpha|=|\beta|=m} \int_{\Omega} D^{\alpha} \phi_{i} A_{i j}^{\alpha \beta}(x / \varepsilon) D^{\beta} w_{\varepsilon j}\right| \\
& \left.\leq C\left\{\left\|\nabla^{m} \phi\right\|_{L^{2}\left(\Omega_{4 \varepsilon}\right)}\left\|\nabla^{m} u_{0}\right\|_{L^{2}\left(\Omega_{5 \varepsilon}\right)}+\varepsilon\left\|\nabla^{m+1} u_{0}\right\|_{L^{2}\left(\Omega^{2 \varepsilon} ; \vartheta\right)}\left\|\nabla^{m} \phi\right\|_{L^{2}\left(\Omega^{2 \varepsilon} ; \vartheta-1\right.}\right)\right\} .
\end{aligned}
$$

Taking $\vartheta=1, \phi=w_{\varepsilon}$ and using the ellipticity condition (1.2), we obtain that

$$
\left\|\nabla^{m} w_{\varepsilon}\right\|_{H_{0}^{m}(\Omega)} \leq C\left\{\left\|\nabla^{m} u_{0}\right\|_{L^{2}\left(\Omega_{5 \varepsilon}\right)}+\varepsilon\left\|\nabla^{m+1} u_{0}\right\|_{L^{2}\left(\Omega^{2 \varepsilon}\right)}\right\},
$$

from which and (3.3), (3.4), we obtain (3.22) immediately.

We are now prepared to prove Theorem 1.4.

Proof of Theorem 1.4 We only provide the details for (1.14), as the proof of (1.15) is similar. By scaling, we may assume that

$$
\|\dot{g}\|_{W A^{m, 2}(\partial \Omega)}+\|f\|_{H^{-m+1}(\Omega)}=1 .
$$

For any fixed $F \in H^{-m+1}\left(\Omega ; \mathbb{R}^{n}\right)$, let $\psi_{\varepsilon} \in H_{0}^{m}\left(\Omega ; \mathbb{R}^{n}\right)$ be the weak solution to the Dirichlet problem

$$
\begin{cases}\mathcal{L}_{\varepsilon}^{*} \psi_{\varepsilon}=F & \text { in } \Omega, \\ \operatorname{Tr}\left(D^{\gamma} \psi_{\varepsilon}\right)=0 & \text { on } \partial \Omega \text { for } 0 \leq|\gamma| \leq m-1,\end{cases}
$$


and let $\psi_{0} \in H_{0}^{m}\left(\Omega ; \mathbb{R}^{n}\right)$ be the weak solution to the homogenized problem

$$
\begin{cases}\mathcal{L}_{0}^{*} \psi_{0}=F & \text { in } \Omega \\ \operatorname{Tr}\left(D^{\gamma} \psi_{0}\right)=0 & \text { on } \partial \Omega \text { for } 0 \leq|\gamma| \leq m-1\end{cases}
$$

Here, $\mathcal{L}_{\varepsilon}^{*}$ and $\mathcal{L}_{0}^{*}$ are the adjoint operators of $\mathcal{L}_{\varepsilon}$ and $\mathcal{L}_{0}$, respectively. Let $0 \leq \widetilde{\rho}_{\varepsilon} \leq 1$ be a function in $C_{c}^{\infty}(\Omega)$ with $\operatorname{supp}\left(\widetilde{\rho}_{\varepsilon}\right) \subset \Omega^{6 \varepsilon}, \widetilde{\rho}_{\varepsilon}=1$ on $\Omega^{8 \varepsilon}$ and $\left|\nabla^{m} \widetilde{\rho}_{\varepsilon}\right| \leq C \varepsilon^{-m}$. Set

$$
\Psi_{\varepsilon}=\psi_{\varepsilon}-\psi_{0}-\varepsilon^{m} \sum_{|\gamma|=m} \chi^{* \gamma}(x / \varepsilon) S_{\varepsilon}^{2}\left(D^{\gamma} \psi_{0}\right) \tilde{\rho}_{\varepsilon} .
$$

It satisfies the same properties as $w_{\varepsilon}$, since $A^{*}$ satisfies the same properties as $A$. Note that $w_{\varepsilon} \in H_{0}^{m}\left(\Omega ; \mathbb{R}^{n}\right)$, and we deduce that

$$
\begin{aligned}
\left\langle F, w_{\varepsilon}\right\rangle_{H^{-m+1}(\Omega) \times H_{0}^{m-1}(\Omega)=} & \sum_{|\alpha|=|\beta|=m} \int_{\Omega} A^{\beta \alpha}(x / \varepsilon) D^{\alpha} w_{\varepsilon} D^{\beta} \Psi_{\varepsilon} \\
& +\sum_{|\alpha|=|\beta|=m} \int_{\Omega} A^{\beta \alpha}(x / \varepsilon) D^{\alpha} w_{\varepsilon} D^{\beta} \psi_{0} \\
& +\sum_{|\alpha|=|\beta|=m} \int_{\Omega} A^{\beta \alpha}(x / \varepsilon) D^{\alpha} w_{\varepsilon} D^{\beta} \\
& \times\left\{\sum_{|\gamma|=m} \varepsilon^{m} \chi^{* \gamma}(x / \varepsilon) S_{\varepsilon}^{2}\left(D^{\gamma} \psi_{0}\right) \tilde{\rho}_{\varepsilon}\right\} \\
\doteq & J_{1}+J_{2}+J_{3} .
\end{aligned}
$$

By (3.22), we obtain that

$$
\left|J_{1}\right| \leq C\left\|w_{\varepsilon}\right\|_{H_{0}^{m}(\Omega)}\left\|\Psi_{\varepsilon}\right\|_{H_{0}^{m}(\Omega)} \leq C_{\nu} \varepsilon^{1-2 v}\|F\|_{H^{-m+1}(\Omega)} .
$$

Using (3.24) and taking $\vartheta(x)=\delta(x)$, we have

$$
\begin{aligned}
\left|J_{2}\right| \leq & C \varepsilon\left\|\nabla^{m} \psi_{0}\right\|_{L^{2}\left(\Omega^{2 \varepsilon} ; \delta^{-1}\right)}\left\|\nabla^{m+1} u_{0}\right\|_{L^{2}\left(\Omega^{2 \varepsilon} ; \delta\right)} \\
& +C\left\|\nabla^{m} u_{0}\right\|_{L^{2}\left(\Omega_{5 \varepsilon}\right)}\left\|\nabla^{m} \psi_{0}\right\|_{L^{2}\left(\Omega_{4 \varepsilon}\right)} .
\end{aligned}
$$

By (3.3) (note that $\psi_{0}$ also satisfies (3.3)), we get

$$
\left\|\nabla^{m} u_{0}\right\|_{L^{2}\left(\Omega_{5 \varepsilon}\right)}\left\|\nabla^{m} \psi_{0}\right\|_{L^{2}\left(\Omega_{4 \varepsilon}\right)} \leq C_{\nu} \varepsilon^{1-2 v}\|F\|_{H^{-m+1}(\Omega)} .
$$

Furthermore, taking (3.5) and (3.6) into consideration, we conclude from (3.26) that

$$
\left|J_{2}\right| \leq C_{\nu} \varepsilon^{1-2 v}\|F\|_{H^{-m+1}(\Omega)} .
$$

We now turn to $J_{3}$. By (3.24), we obtain that

$$
\begin{aligned}
\left|J_{3}\right| \leq & C \varepsilon \sum_{|\gamma|=m}\left\|\nabla^{m+1} u_{0}\right\|_{L^{2}\left(\Omega^{2 \varepsilon} ; \delta\right)} \varepsilon^{m}\left\|\nabla^{m}\left\{\chi^{* \gamma}(x / \varepsilon) S_{\varepsilon}^{2}\left(D^{\gamma} \psi_{0}\right) \widetilde{\rho}_{\varepsilon}\right\}\right\|_{L^{2}\left(\Omega^{2 \varepsilon} ; \delta^{-1}\right)} \\
& +C \sum_{|\gamma|=m} \varepsilon^{m}\left\|\nabla^{m} u_{0}\right\|_{L^{2}\left(\Omega_{5 \varepsilon}\right)}\left\|\nabla^{m}\left\{\chi^{* \gamma}(x / \varepsilon) S_{\varepsilon}^{2}\left(D^{\gamma} \psi_{0}\right) \widetilde{\rho}_{\varepsilon}\right\}\right\|_{L^{2}\left(\Omega_{4 \varepsilon}\right)}
\end{aligned}
$$


where the last term is zero by the definition of $\tilde{\rho}_{\varepsilon}$. To estimate the first term, we note that

$$
\begin{aligned}
\varepsilon^{m} \| & D^{\beta}\left\{\chi^{* \gamma}(x / \varepsilon) S_{\varepsilon}^{2}\left(D^{\gamma} \psi_{0}\right) \tilde{\rho}_{\varepsilon}\right\} \|_{L^{2}\left(\Omega^{2 \varepsilon} ; \delta^{-1}\right)} \\
\leq & C\left\|\left(D^{\beta} \chi^{* \gamma}\right)(x / \varepsilon) S_{\varepsilon}^{2}\left(D^{\gamma} \psi_{0}\right) \widetilde{\rho}_{\varepsilon}\right\|_{L^{2}\left(\Omega^{2 \varepsilon} ; \delta^{-1}\right)} \\
& +C \varepsilon^{m}\left\|\chi^{* \gamma}(x / \varepsilon) S_{\varepsilon}^{2}\left(D^{\beta+\gamma} \psi_{0}\right) \widetilde{\rho}_{\varepsilon}\right\|_{L^{2}\left(\Omega^{2 \varepsilon} ; \delta^{-1}\right)} \\
& +C \varepsilon^{m}\left\|\chi^{* \gamma}(x / \varepsilon) S_{\varepsilon}^{2}\left(D^{\gamma} \psi_{0}\right) D^{\beta} \widetilde{\rho}_{\varepsilon}\right\|_{L^{2}\left(\Omega^{2 \varepsilon} ; \delta^{-1}\right)} \\
& +C \sum_{|\zeta+\eta+\xi|=m} \varepsilon^{|\eta|+|\xi|}\left\|\left(D^{\zeta} \chi^{* \gamma}\right)(x / \varepsilon) S_{\varepsilon}^{2}\left(D^{\gamma+\eta} \psi_{0}\right) D^{\xi} \widetilde{\rho}_{\varepsilon}\right\|_{L^{2}\left(\Omega^{2 \varepsilon} ; \delta^{-1}\right)} \\
& \doteq J_{31}+J_{32}+J_{33}+J_{34},
\end{aligned}
$$

for all multi-indexes $\beta, \gamma$ with length $m$. By Lemmas 2.1 and 3.2, we obtain that

$$
\begin{aligned}
& J_{31} \leq C\left\|S_{\varepsilon}\left(\nabla^{m} \psi_{0}\right)\right\|_{L^{2}\left(\Omega^{6 \varepsilon} ; \delta^{-1}\right)} \leq C\left\|\nabla^{m} \psi_{0}\right\|_{L^{2}\left(\Omega^{5 \varepsilon} ; \delta^{-1}\right)} \leq C_{\nu} \varepsilon^{-v}\|F\|_{H^{-m+1}(\Omega)}, \\
& J_{33} \leq C\left\|S_{\varepsilon}\left(\nabla^{m} \psi_{0}\right)\right\|_{L^{2}\left(\Omega_{9 \varepsilon} \backslash \Omega_{4 \varepsilon} ; \delta^{-1}\right)} \leq C_{\nu} \varepsilon^{-v}\|F\|_{H^{-m+1}(\Omega)} .
\end{aligned}
$$

Furthermore, by Lemmas 2.1, 2.2 and 3.2, we see that

$$
\begin{aligned}
& J_{32} \leq C \varepsilon^{m}\left\|S_{\varepsilon}\left(\nabla^{2 m} \psi_{0}\right)\right\|_{L^{2}\left(\Omega^{4 \varepsilon} ; \delta^{-1}\right)} \leq C_{\nu} \varepsilon^{-v}\|F\|_{H^{-m+1}(\Omega)}, \\
& J_{34}=C \sum_{\substack{k_{1}+k_{2}+k_{3}=m \\
1 \leq k_{i}, i=1,2,3}} \varepsilon^{k_{2}+k_{3}}\left\|\left(\nabla^{k_{1}} \chi^{*}\right)(x / \varepsilon) S_{\varepsilon}^{2}\left(\nabla^{k_{2}+m} \psi_{0}\right) \nabla^{k_{3}} \widetilde{\rho}_{\varepsilon}\right\|_{L^{2}\left(\Omega_{8 \varepsilon} \backslash \Omega_{6 \varepsilon} ; \delta^{-1}\right)} \\
& \leq C \sum_{1 \leq k_{2} \leq m-2} \varepsilon^{k_{2}}\left\|S_{\varepsilon}\left(\nabla^{k_{2}+m} \psi_{0}\right)\right\|_{L^{2}\left(\Omega_{9_{\varepsilon}} \backslash \Omega_{5 \varepsilon} ; \delta^{-1}\right)} \\
& \leq C_{\nu} \varepsilon^{-v}\|F\|_{H^{-m+1}(\Omega)} \text {. }
\end{aligned}
$$

Taking the estimates on $J_{31}, J_{32}, J_{33}, J_{34}$ into (3.27), and using (3.6), we obtain that

$$
\left|J_{3}\right| \leq C_{\nu} \varepsilon^{1-2 v}\|F\|_{H^{-m+1}(\Omega)} .
$$

In view of the estimates on $J_{1}, J_{2}, J_{3}$ and (3.25), we have proved that

$$
\left|\left\langle F, w_{\varepsilon}\right\rangle_{H^{-m+1}(\Omega) \times H_{0}^{m-1}(\Omega)}\right| \leq C_{\nu} \varepsilon^{1-2 v}\|F\|_{H^{-m+1}(\Omega)},
$$

which, combined with the following estimate

$$
\left\|\varepsilon^{m} \sum_{|\gamma|=m} \chi^{\gamma}(x / \varepsilon) S_{\varepsilon}^{2}\left(D^{\gamma} u_{0}\right) \rho_{\varepsilon}\right\|_{H_{0}^{m-1}(\Omega)} \leq C \varepsilon
$$

gives (1.14). The proof is complete.

Remark 3.1 Part of our motivation for the proof of (1.14) is the finding that $u_{0}$ satisfying certain weighted estimates such as (3.3)-(3.6), which give a proper control on the solution $u_{0}$ in $\Omega^{\varepsilon}$ and $\Omega_{\varepsilon}$. This also inspires us to modify the duality method with weight $\delta(x)$. We mention that weight functions have been used previously in $[22,42]$ to derive the suboptimal $O(\varepsilon \ln (1 / \varepsilon))$ convergence rate for second-order elliptic systems with symmetric coefficients. Our consideration on the suboptimal convergence rate is also in debt to these works. 


\section{Uniform $C^{m-1, \lambda}$ estimates}

In this section, we consider the uniform boundary $C^{m-1, \lambda}, 0<\lambda<1$, estimate of $u_{\varepsilon}$ in $C^{1}$ domains. Throughout the section, we always assume that $A$ satisfies (1.2) and (1.3). Recall that locally the boundary of a $C^{1}$ domain is the graph of a $C^{1}$ function; we thus restrict our considerations to equations on $\left(D_{r}, \Delta_{r}\right)$ defined in (1.5) with the defining function satisfying (1.4). Let

$$
\mathfrak{P}_{k}=\left\{\left(P_{k}^{1}, P_{k}^{2}, \ldots, P_{k}^{n}\right) \mid P_{k}^{i}, 1 \leq i \leq n, \text { are polynomials of degree } k\right\} .
$$

Let $u_{\varepsilon} \in H^{m}\left(D_{2 r} ; \mathbb{R}^{n}\right)$ be a weak solution to

$$
\mathcal{L}_{\varepsilon} u_{\varepsilon}=F \quad \text { in } D_{2 r}, \quad \operatorname{Tr}\left(D^{\gamma} u_{\varepsilon}\right)=D^{\gamma} G \text { on } \Delta_{2 r} \quad \text { for } 0 \leq|\gamma| \leq m-1,
$$

where $G \in C^{m-1,1}\left(D_{2 r} ; \mathbb{R}^{n}\right), F \in L^{p}\left(D_{2 r} ; \mathbb{R}^{n}\right)$ with $p>\max \{1,2 d /(d+2 m-2)\}$. Define

$$
\begin{aligned}
\Phi_{\lambda}\left(r, u_{\varepsilon}\right)= & \frac{1}{r^{m-1+\lambda}} \inf _{P_{m-1} \in \mathfrak{P}_{m-1}}\left\{\left(f_{D_{r}}\left|u_{\varepsilon}-P_{m-1}\right|^{2}\right)^{1 / 2}+r^{2 m}\left(f_{D_{r}}|F|^{p}\right)^{1 / p}\right. \\
& \left.+\sum_{j=0}^{m} r^{j}\left\|\nabla^{j}\left(G-P_{m-1}\right)\right\|_{L^{\infty}\left(D_{r}\right)}\right\}, \quad 0<\lambda<1 .
\end{aligned}
$$

Lemma 4.1 Let $0<\varepsilon \leq r \leq 1$ and $\Phi_{\lambda}\left(r, u_{\varepsilon}\right)$ be defined as above. There exists $u_{0} \in$ $H^{m}\left(D_{r} ; \mathbb{R}^{n}\right)$ such that $\mathcal{L}_{0} u_{0}=F$ in $D_{r}, \operatorname{Tr}\left(D^{\gamma} u_{0}\right)=D^{\gamma} G$ on $\Delta_{r}$ for $0 \leq|\gamma| \leq m-1$, and

$$
\left(f_{D_{r}}\left|u_{\varepsilon}-u_{0}\right|^{2}\right)^{1 / 2} \leq C r^{m-1+\lambda}\left(\frac{\varepsilon}{r}\right)^{1 / 4} \Phi_{\lambda}\left(2 r, u_{\varepsilon}\right),
$$

where $C$ depends only on $d, n, m, p, \mu$ and $M$ in (1.4).

Proof Let us first assume that $r=1$. By Caccioppoli's inequality (see Remark 2.1), we have

$$
\left\|u_{\varepsilon}\right\|_{H^{m}\left(D_{3 / 2}\right)} \leq C\left\{\left\|u_{\varepsilon}\right\|_{L^{2}\left(D_{2}\right)}+\|F\|_{L^{p}\left(D_{2}\right)}+\sum_{j=0}^{m}\left\|\nabla^{j} G\right\|_{L^{2}\left(D_{2}\right)}\right\},
$$

for $p>\max \{1,2 d /(d+2 m)\}$. By the co-area formula, there exists $t \in[5 / 4,3 / 2]$ such that

$$
\left\|u_{\varepsilon}\right\|_{H^{m}\left(\partial D_{t} \backslash \Delta_{2}\right)} \leq C\left\{\left\|u_{\varepsilon}\right\|_{L^{2}\left(D_{2}\right)}+\|F\|_{L^{p}\left(D_{2}\right)}+\sum_{j=0}^{m}\left\|\nabla^{j} G\right\|_{L^{2}\left(D_{2}\right)}\right\},
$$

where $C$ depends only on $d, n, m, \mu$. Now let $u_{0}$ be the weak solution to

$$
\mathcal{L}_{0} u_{0}=F \text { in } D_{t}, \quad \operatorname{Tr}\left(D^{\gamma} u_{0}\right)=\operatorname{Tr}\left(D^{\gamma} u_{\varepsilon}\right) \text { on } \partial D_{t} .
$$

Note that $F \in L^{p} \hookrightarrow H^{-m+1}$ when $p>\max \{2 d /(d+2 m-2), 1\}$. As a consequence of (1.14) in Theorem 1.4, we have

$$
\left\|u_{\varepsilon}-u_{0}\right\|_{L^{2}\left(D_{t}\right)} \leq C \varepsilon^{1 / 4}\left\{\left\|u_{\varepsilon}\right\|_{H^{m}\left(\partial D_{t}\right)}+\|F\|_{L^{p}\left(D_{2}\right)}\right\},
$$

where $C$ depends only on $d, n, m, \mu$ and $M$ in (1.4). This, together with (4.5), yields $\left\|u_{\varepsilon}-u_{0}\right\|_{L^{2}\left(D_{1}\right)} \leq\left\|u_{\varepsilon}-u_{0}\right\|_{L^{2}\left(D_{t}\right)} \leq C \varepsilon^{1 / 4}\left\{\left\|u_{\varepsilon}\right\|_{L^{2}\left(D_{2}\right)}+\|F\|_{L^{p}\left(D_{2}\right)}+\|G\|_{C^{m-1,1}\left(D_{2}\right)}\right\}$, 
for $p>\max \{1,2 d /(d+2 m-2)\}$.

We now perform scaling for general $\varepsilon \leq r<1$. Set

$$
v_{\varepsilon}(x)=u_{\varepsilon}(r x), \quad \widetilde{G}(x)=G(r x), \quad \widetilde{F}(x)=r^{2 m} F(r x) .
$$

By (4.1), we know that $\mathcal{L}_{\frac{\varepsilon}{r}} v_{\varepsilon}=\widetilde{F}(x)$ in $\widetilde{D}_{2}$, and $\operatorname{Tr}\left(D^{\gamma} v_{\varepsilon}\right)=D^{\gamma} \widetilde{G}(x)$ on $\widetilde{\Delta}_{2}$ for $0 \leq|\gamma| \leq$ $m-1$, where

$$
\begin{aligned}
& \widetilde{D}_{2}=\left\{\left(x^{\prime}, x_{d}\right) \in \mathbb{R}^{d}:\left|x^{\prime}\right|<2 \text { and } \psi_{r}\left(x^{\prime}\right)<x_{d}<\psi_{r}\left(x^{\prime}\right)+2\right\}, \\
& \widetilde{\Delta}_{2}=\left\{\left(x^{\prime}, \psi_{r}\left(x^{\prime}\right)\right) \in \mathbb{R}^{d}:\left|x^{\prime}\right|<2\right\}, \text { with } \psi_{r}\left(x^{\prime}\right)=r^{-1} \psi\left(r x^{\prime}\right) .
\end{aligned}
$$

Thanks to (4.6), there exists $v_{0}$ with $\mathcal{L}_{0} v_{0}=\widetilde{F}(x)$ in $\widetilde{D}_{1}, \operatorname{Tr}\left(D^{\gamma} v_{0}\right)=\operatorname{Tr}\left(D^{\gamma} v_{\varepsilon}\right)$ on $\partial \widetilde{D}_{1}$ for $0 \leq|\gamma| \leq m-1$, such that

$$
\left\|v_{\varepsilon}-v_{0}\right\|_{L^{2}\left(\widetilde{D}_{1}\right)} \leq C\left(\frac{\varepsilon}{r}\right)^{1 / 4}\left\{\left\|v_{\varepsilon}\right\|_{L^{2}\left(\widetilde{D}_{2}\right)}+\|\widetilde{F}(x)\|_{L^{p}\left(\widetilde{D}_{2}\right)}+\|\widetilde{G}\|_{C^{m-1,1}\left(\widetilde{D}_{2}\right)}\right\} .
$$

Setting $u_{0}(x)=v_{0}(x / r)$, we then obtain by the change of variables,

$$
\begin{aligned}
\left(f_{D_{r}}\left|u_{\varepsilon}-u_{0}\right|^{2}\right)^{1 / 2} \leq & C\left(\frac{\varepsilon}{r}\right)^{1 / 4}\left\{\left(f_{D_{2 r}}\left|u_{\varepsilon}\right|^{2}\right)^{1 / 2}+r^{2 m}\left(f_{D_{2 r}}|F|^{p}\right)^{1 / p}\right. \\
& \left.+\sum_{j=0}^{m} r^{j}\left\|\nabla^{j} G\right\|_{L^{\infty}\left(D_{2 r}\right)}\right\} .
\end{aligned}
$$

Note that the above inequality still hold if we subtract a polynomial $P_{m-1} \in \mathfrak{P}_{m-1}$ from $u_{\varepsilon}, u_{0}$ and $G$ simultaneously. This gives (4.3) by taking the infimum with respect to $P_{m-1}$.

Lemma 4.2 For $0<\varepsilon \leq r \leq 1$, let $u_{0} \in H^{m}\left(D_{2} ; \mathbb{R}^{n}\right)$ be a weak solution to

$$
\mathcal{L}_{0} u_{0}=F \quad \text { in } D_{2}, \quad \operatorname{Tr}\left(D^{\gamma} u_{0}\right)=D^{\gamma} G \quad \text { on } \Delta_{2} \quad \text { for } 0 \leq|\gamma| \leq m-1,
$$

where $G \in C^{m-1,1}\left(D_{2} ; \mathbb{R}^{n}\right), F \in L^{p}\left(D_{2} ; \mathbb{R}^{n}\right)$ with $p>\max \{d /(m+1), 2 d /(d+2 m-$ $2), 1\}$. Then, for any $0<\lambda<\min \{m+1-d / p, 1\}$, there exist $\lambda_{0}<\min \{m+1-d / p, 1\}$ and a constant $C$ depending only on $d, n, m, p, \mu, M$ and $\tau(t)$ in (1.4), such that

$$
\Phi_{\lambda}\left(\delta r ; u_{0}\right) \leq C \delta^{\lambda_{0}-\lambda} \Phi_{\lambda}\left(r ; u_{0}\right) \text { for } 0<\delta<1 / 4 \text {. }
$$

Proof By rescaling, we assume that $r=1$. For $0<\lambda<\min \{m+1-d / p, 1\}$, fix $\lambda_{0}$ such that $\lambda<\lambda_{0}<\min \{m+1-d / p, 1\}$. Set $P_{m-1}$ in (4.2) as

$$
P_{m-1}(x)=\sum_{|\alpha|=0}^{m-1} \frac{1}{\alpha !} D^{\alpha} u_{0}(0) x^{\alpha}=\sum_{|\alpha|=0}^{m-1} \frac{1}{\alpha !} D^{\alpha} G(0) x^{\alpha} .
$$

It is not difficult to find that

$$
\begin{aligned}
\Phi_{\lambda}\left(\delta, u_{0}\right) \leq & C \delta^{\lambda_{0}-\lambda}\left\|u_{0}\right\|_{C^{m-1, \lambda_{0}}\left(D_{\delta}\right)} \\
& +\delta^{m+1-\lambda-d / p}\left(f_{D_{1}}|F|^{p}\right)^{1 / p}+\delta^{1-\lambda} \sum_{j=0}^{m}\left\|\nabla^{j} G\right\|_{L^{\infty}\left(D_{1}\right)}
\end{aligned}
$$


for any $0<\delta<1 / 4$. Observe that when $p>\max \{d /(m+1), 1\}, L^{p}\left(D_{2} ; \mathbb{R}^{n}\right) \hookrightarrow$ $W^{-m, q}\left(D_{2} ; \mathbb{R}^{n}\right)$ for some $q>d$. Combining the $C^{m-1, \lambda_{0}}$ estimate for higher-order elliptic systems with constant coefficients in $C^{1}$ domains (see e.g., $[10,12]$ ) and a localization argument (see e.g., the proof of Corollary 5.1), we have

$$
\begin{aligned}
\left\|u_{0}\right\|_{C^{m-1, \lambda_{0}\left(D_{\delta}\right)}} & \leq C\left\|u_{0}\right\|_{C^{m-1, \lambda_{0}}\left(D_{1 / 4}\right)} \\
& \leq C\left\{\left(f_{D_{1}}\left|u_{0}\right|^{2}\right)^{1 / 2}+\left(f_{D_{1}}|F|^{p}\right)^{1 / p}+\sum_{j=0}^{m}\left\|\nabla^{j} G\right\|_{L^{\infty}\left(D_{1}\right)}\right\} .
\end{aligned}
$$

Taking (4.9) into (4.8), we derive that

$$
\Phi_{\lambda}\left(\delta, u_{0}\right) \leq C \delta^{\lambda_{0}-\lambda}\left\{\left(f_{D_{1}}\left|u_{0}\right|^{2}\right)^{1 / 2}+\left(f_{D_{1}}|F|^{p}\right)^{1 / p}+\sum_{j=0}^{m}\left\|\nabla^{j} G\right\|_{L^{\infty}\left(D_{1}\right)}\right\} .
$$

Substituting $u_{0}, G$ by $u_{0}-P_{m-1}$ and $G-P_{m-1}$, respectively, and taking the infimum with respect to $P_{m-1} \in \mathfrak{P}_{m-1}$, we obtain (4.7) immediately.

Lemma 4.3 For $0<\varepsilon \leq r \leq 1 / 2$, let $\Phi_{\lambda}\left(r, u_{\varepsilon}\right)$ be defined as in (4.2). Then, there exists $\delta \in(0,1 / 4)$ depending only on $d, n, m, p, \lambda, \mu, M$ and $\tau(t)$ in (1.4), such that

$$
\Phi_{\lambda}\left(\delta r ; u_{\varepsilon}\right) \leq \frac{1}{2} \Phi_{\lambda}\left(2 r ; u_{\varepsilon}\right)+C\left(\frac{\varepsilon}{r}\right)^{1 / 4} \Phi_{\lambda}\left(2 r ; u_{\varepsilon}\right),
$$

where $C$ depends only on $d, n, m, p, \lambda, \mu, M$ and $\tau(t)$ in (1.4).

Proof By the definition, it is easy to find that

$$
\begin{aligned}
\Phi_{\lambda}\left(\delta r ; u_{\varepsilon}\right) \leq & \Phi_{\lambda}\left(\delta r ; u_{0}\right)+\frac{1}{(\delta r)^{m-1+\lambda}}\left(f_{D_{\delta r}}\left|u_{\varepsilon}-u_{0}\right|^{2}\right)^{1 / 2} \\
\leq & C \delta^{\lambda_{0}-\lambda} \Phi_{\lambda}\left(r ; u_{0}\right)+\frac{1}{(\delta r)^{m-1+\lambda}}\left(f_{D_{\delta r}}\left|u_{\varepsilon}-u_{0}\right|^{2}\right)^{1 / 2} \\
\leq & C \delta^{\lambda_{0}-\lambda} \Phi_{\lambda}\left(r ; u_{\varepsilon}\right)+\frac{C \delta^{\lambda_{0}-\lambda}}{r^{m-1+\lambda}}\left(f_{D_{r}}\left|u_{\varepsilon}-u_{0}\right|^{2}\right)^{1 / 2} \\
& +\frac{1}{(\delta r)^{m-1+\lambda}}\left(f_{D_{\delta r}}\left|u_{\varepsilon}-u_{0}\right|^{2}\right)^{1 / 2} \\
\leq & C \delta^{\lambda_{0}-\lambda} \Phi_{\lambda}\left(2 r ; u_{\varepsilon}\right)+\frac{C_{\delta}}{r^{m-1+\lambda}}\left(f_{D_{r}}\left|u_{\varepsilon}-u_{0}\right|^{2}\right)^{1 / 2} .
\end{aligned}
$$

Taking $\delta$ small enough such that $C \delta^{\lambda_{0}-\lambda}<1 / 2$, and then using Lemma 4.1, we obtain (4.10) directly.

Proof of Theorem 1.1 We only need to consider the case $\varepsilon \leq r<1 / 4$, since the estimate (1.6) is trivial when $1 / 4 \leq r \leq 1$, following directly from Caccioppoli's inequality. Thanks to Lemma 4.3 , we can take $N_{0}$ large enough such that

$$
\Phi_{\lambda}\left(\delta r ; u_{\varepsilon}\right) \leq \frac{1}{2} \Phi_{\lambda}\left(2 r ; u_{\varepsilon}\right)+C\left(\frac{1}{N_{0}}\right)^{1 / 4} \Phi_{\lambda}\left(2 r ; u_{\varepsilon}\right) \leq \Phi_{\lambda}\left(2 r ; u_{\varepsilon}\right),
$$

for $r \geq N_{0} \varepsilon$, where $\delta$ given by Lemma 4.3 is fixed. Hence, by iteration we have

$$
\Phi_{\lambda}\left(r ; u_{\varepsilon}\right) \leq C \Phi_{\lambda}\left(1 ; u_{\varepsilon}\right) \quad \text { for } r \in\left[N_{0} \varepsilon, 1 / 2\right) .
$$


On the other hand, for $\varepsilon \leq r<N_{0} \varepsilon$, it is obvious that

$$
\Phi_{\lambda}\left(r ; u_{\varepsilon}\right) \leq C \Phi_{\lambda}\left(N_{0} \varepsilon ; u_{\varepsilon}\right) \leq C \Phi_{\lambda}\left(1 ; u_{\varepsilon}\right),
$$

where $C$ depends on $N_{0}$. This, together with (4.12), gives

$$
\Phi_{\lambda}\left(r ; u_{\varepsilon}\right) \leq C \Phi_{\lambda}\left(1 ; u_{\varepsilon}\right) \quad \text { for } \varepsilon \leq r \leq 1 / 2 .
$$

By Caccioppoli's inequality, we deduce that

$$
\begin{aligned}
\left(f_{D_{r}}\left|\nabla^{m}\left(u_{\varepsilon}-P_{m-1}\right)\right|^{2}\right)^{1 / 2} \leq & C r^{-m} \inf _{P_{m-1} \in \mathfrak{P}_{m-1}}\left\{\left(f_{D_{2 r}}\left|u_{\varepsilon}-P_{m-1}\right|^{2}\right)^{1 / 2}\right. \\
& +r^{2 m}\left(f_{D_{2 r}}|F|^{p}\right)^{1 / p} \\
& \left.+\sum_{j=0}^{m} r^{j}\left(f_{D_{2 r}}\left|\nabla^{j}\left(G-P_{m-1}\right)\right|^{2}\right)^{1 / 2}\right\} \\
= & C r^{\lambda-1} \Phi_{\lambda}\left(2 r, u_{\varepsilon}\right) \leq C r^{\lambda-1} \Phi_{\lambda}\left(1, u_{\varepsilon}\right) \\
\leq & C r^{\lambda-1}\left\{\left(f_{D_{1}}\left|u_{\varepsilon}\right|^{2}\right)^{1 / 2}\right. \\
& \left.+\left(f_{D_{1}}|F|^{p}\right)^{1 / p}+\sum_{j=0}^{m}\left\|\nabla^{j} G\right\|_{L^{\infty}\left(D_{1}\right)}\right\}
\end{aligned}
$$

for all $\varepsilon \leq r<1 / 2$ and any $P_{m-1} \in \mathfrak{P}_{m-1}$, which is exactly (1.6).

Corollary 4.1 In addition to the assumptions of Theorem 1.1, if $A \in V M O\left(\mathbb{R}^{d}\right)$, then for any $0<\lambda<\min \{m+1-d / p, 1\}$,

$$
\left\|u_{\varepsilon}\right\|_{C^{m-1, \lambda}\left(D_{1 / 4}\right)} \leq C\left\{\left(f_{D_{1}}\left|u_{\varepsilon}\right|^{2}\right)^{1 / 2}+\left(f_{D_{1}}|F|^{p}\right)^{1 / p}+\|G\|_{C^{m-1,1}\left(D_{1}\right)}\right\},
$$

where $C$ depends only on $d, n, m, p, \mu$ as well as $M, \tau(t)$ in (1.4) and $\varrho(t)$ in (1.7).

Proof It is enough to assume $0<\varepsilon<1 / 2$, as the other case is trivial. Setting

$$
v_{\varepsilon}(x)=u_{\varepsilon}(\varepsilon x), \quad \widetilde{F}(x)=\varepsilon^{2 m} F(\varepsilon x), \quad \widetilde{G}(x)=G(\varepsilon x),
$$

then $v_{\varepsilon}$ satisfies

$$
\mathcal{L}_{1} v_{\varepsilon}=\widetilde{F}(x) \quad \text { in } D_{1}, \quad \operatorname{Tr}\left(D^{\gamma} v_{\varepsilon}\right)=D^{\gamma} \widetilde{G}(x) \quad \text { on } \Delta_{1} \quad \text { for } 0 \leq|\gamma| \leq m-1 .
$$

By $C^{m-1, \lambda}$ estimates of operator $\mathcal{L}_{1}$ in $C^{1}$ domains [10,12] and a localization argument, we have for any $0<\lambda<\min \{m+1-d / p, 1\}$ and $0<s<1 / 2$,

$$
\left(f_{D_{s}}\left|\nabla^{m} v_{\varepsilon}\right|^{2}\right)^{1 / 2} \leq C s^{\lambda-1}\left\{\left(f_{D_{1}}\left|v_{\varepsilon}\right|^{2}\right)^{1 / 2}+\left(f_{D_{1}}|\widetilde{F}|^{p}\right)^{1 / p}+\sum_{j=0}^{m}\left\|\nabla^{j} \widetilde{G}\right\|_{L^{\infty}\left(D_{1}\right)}\right\} .
$$

By the change of variables, we obtain for $0<r<\varepsilon / 2$,

$$
\begin{aligned}
\left(f_{D_{r}}\left|\nabla^{m} u_{\varepsilon}\right|^{2}\right)^{1 / 2} \leq & C\left(\frac{r}{\varepsilon}\right)^{\lambda-1} \frac{1}{\varepsilon^{m}}\left\{\left(f_{D_{\varepsilon}}\left|u_{\varepsilon}\right|^{2}\right)^{1 / 2}\right. \\
& \left.+\varepsilon^{2 m}\left(f_{D_{\varepsilon}}|F|^{p}\right)^{1 / p}+\sum_{j=0}^{m} \varepsilon^{j}\left\|\nabla^{j} G\right\|_{L^{\infty}\left(D_{\varepsilon}\right)}\right\} .
\end{aligned}
$$


Subtracting $P_{m-1}$ from $u_{\varepsilon}$ and $G$ simultaneously, and taking (4.13) in consideration, we obtain that

$$
\left(f_{D_{r}}\left|\nabla^{m} u_{\varepsilon}\right|^{2}\right)^{1 / 2} \leq C r^{\lambda-1}\left\{\left(f_{D_{1}}\left|u_{\varepsilon}\right|^{2}\right)^{1 / 2}+\left(f_{D_{1}}|F|^{p}\right)^{1 / p}+\sum_{j=0}^{m}\left\|\nabla^{j} G\right\|_{L^{\infty}\left(D_{1}\right)}\right\}
$$

for any $0<r \leq \varepsilon$. In view of (1.6), we know that (4.18) holds for $0 \leq r<1 / 2$. Combining (4.18) with similar interior $C^{m-1, \lambda}$ estimate in [28, Corollary 5.1], we obtain that

$$
\begin{aligned}
\left(f_{B(x, r) \cap D_{1 / 4}}\left|\nabla^{m} u_{\varepsilon}\right|^{2}\right)^{1 / 2} \leq & C r^{\lambda-1}\left\{\left(f_{D_{1}}\left|u_{\varepsilon}\right|^{2}\right)^{1 / 2}\right. \\
& \left.+\left(f_{D_{1}}|F|^{p}\right)^{1 / p}+\sum_{j=0}^{m}\left\|\nabla^{j} G\right\|_{L^{\infty}\left(D_{1}\right)}\right\}
\end{aligned}
$$

for any $0<r<r_{0}$ ( $r_{0}$ is small) and $x \in D_{1 / 4}$. This gives (4.14) by the Campanato characterization of Hölder spaces.

Remark 4.1 Under the assumptions of Corollary 4.1, if $F, G \equiv 0$ we may use Poincaré's inequality to deduce from (4.17) that

$$
\left(f_{D_{r}}\left|\nabla^{m} u_{\varepsilon}\right|^{2}\right)^{1 / 2} \leq C\left(\frac{r}{\varepsilon}\right)^{\lambda-1}\left(f_{D_{\varepsilon}}\left|\nabla^{m} u_{\varepsilon}\right|^{2}\right)^{1 / 2}
$$

for any $0<r \leq \varepsilon$. This will be used to establish the uniform $W^{m, p}$ estimate in the next section.

\section{Uniform $W^{m, p}$ estimates}

This section is devoted to the uniform $W^{m, p}$ estimate for $u_{\varepsilon}$ in $C^{1}$ domains under the assumption $A \in V M O\left(\mathbb{R}^{d}\right)$.

Lemma 5.1 Assume that $\Omega$ is a bounded $C^{1}$ domain in $\mathbb{R}^{d}$ and the coefficient matrix $A \in$ $V M O\left(\mathbb{R}^{d}\right)$ satisfies (1.2)-(1.3). Let $B\left(x_{0}, r\right), r<r_{0}$, be a ball centered at $x_{0} \in \partial \Omega$ with radius $r$, and $u_{\varepsilon} \in H^{m}\left(B\left(x_{0}, 2 r\right) \cap \Omega ; \mathbb{R}^{n}\right)$ be a weak solution to

$\mathcal{L}_{\varepsilon} u_{\varepsilon}=0$ in $B\left(x_{0}, 2 r\right) \cap \Omega, \quad \operatorname{Tr}\left(D^{\gamma} u_{\varepsilon}\right)=0$ on $B\left(x_{0}, 2 r\right) \cap \partial \Omega$ for $0 \leq|\gamma| \leq m-1$.

Then, for any $2 \leq p<\infty$,

$$
\left(f_{B\left(x_{0}, r\right) \cap \Omega}\left|\nabla^{m} u_{\varepsilon}\right|^{p}\right)^{1 / p} \leq C\left(f_{B\left(x_{0}, 2 r\right) \cap \Omega}\left|\nabla^{m} u_{\varepsilon}\right|^{2}\right)^{1 / 2}
$$

where $C$ depends only on $d, n, m, p, \mu$ as well as $M, \tau(t)$ in (1.4) and $\varrho(t)$ in (1.7).

Proof We only need to consider the case $\varepsilon<\frac{1}{4}$. Since if else $A(x / \varepsilon)$ satisfies (1.7) uniformly, and (5.1) follows from the existing $W^{m, p}$ estimates for higher-order elliptic systems with $V M O$ coefficients, see e.g., $[10,12]$. Also, note that the function $\psi_{r}\left(x^{\prime}\right)=r^{-1} \psi\left(r x^{\prime}\right)$ satisfies condition (1.4) uniformly. We can then fix our considerations on the case $r=1$ by rescaling. By the uniform interior $W^{m, p}$ estimates derived by the authors in [28, Theorem 1.3], we have

$$
\left(f_{B(x, t)}\left|\nabla^{m} u_{\varepsilon}\right|^{p}\right)^{1 / p} \leq C\left(f_{B(x, 2 t)}\left|\nabla^{m} u_{\varepsilon}\right|^{2} \mathrm{~d} x\right)^{1 / 2},
$$


whenever $u_{\varepsilon}$ is a weak solution to $\mathcal{L}_{\varepsilon} u_{\varepsilon}=0$ in $B(x, 2 t)$. Therefore, in view of (1.6) and (4.19), we have for any $0<\lambda<1$ and $y \in B\left(x_{0}, 1\right) \cap \Omega$,

$$
\begin{aligned}
\left(f_{B(y, \delta(y) / 8)}\left|\nabla^{m} u_{\varepsilon}\right|^{p}\right)^{1 / p} & \leq C\left(f_{B(y, \delta(y) / 4)}\left|\nabla^{m} u_{\varepsilon}\right|^{2}\right)^{1 / 2} \\
& \leq C[\delta(y)]^{\lambda-1}\left(\int_{B\left(x_{0}, 2\right) \cap \Omega}\left|\nabla^{m} u_{\varepsilon}\right|^{2}\right)^{1 / 2},
\end{aligned}
$$

where $\delta(y)$ denotes the distance of $y$ to $\partial\left(B\left(x_{0}, 2\right) \cap \Omega\right)$. Fix $\lambda \in(1-1 / p, 1)$ and integrate (5.2) with respect to $y$ in $B\left(x_{0}, 1\right) \cap \Omega$. We obtain that

$$
\int_{B\left(x_{0}, 1\right) \cap \Omega} f_{B(y, \delta(y) / 8)}\left|\nabla^{m} u_{\varepsilon}\right|^{p} \mathrm{~d} x \mathrm{~d} y \leq C\left\|\nabla^{m} u_{\varepsilon}\right\|_{L^{2}\left(B\left(x_{0}, 2\right) \cap \Omega\right)}^{p} .
$$

We then deduce from Fubini's theorem that

$$
\int_{B\left(x_{0}, 1\right) \cap \Omega}\left|\nabla^{m} u_{\varepsilon}(x)\right|^{p} \int_{\left\{y \in B\left(x_{0}, 1\right) \cap \Omega:|x-y|<\delta(y) / 8\right\}} \frac{1}{\delta(y)^{d}} \mathrm{~d} y \mathrm{~d} x \leq C\left\|\nabla^{m} u_{\varepsilon}\right\|_{L^{2}\left(B\left(x_{0}, 2\right) \cap \Omega\right)}^{p} .
$$

Observe that when $|x-y|<\delta(y) / 8$, it holds that

$$
\frac{1}{2} \delta(y) \leq \delta(x) \leq 2 \delta(y) .
$$

We thus conclude that

$$
B\left(x_{0}, 1\right) \cap \Omega \cap B(x, \delta(x) / 16) \subset\left\{y \in B\left(x_{0}, 1\right) \cap \Omega:|x-y|<\delta(y) / 8\right\}
$$

for any $x \in B\left(x_{0}, 1\right) \cap \Omega$. This, together with (5.5), implies that

$$
\int_{\left\{y \in B\left(x_{0}, 1\right) \cap \Omega:|x-y|<\delta(y) / 8\right\}} \frac{1}{\delta(y)^{d}} \mathrm{~d} y \geq C_{0}>0 .
$$

Taking this into (5.4), we obtain (5.1) immediately.

With Lemma 5.1 at our disposal, we are ready to prove Theorem 1.2. The proof is based on a real-variable argument in the following theorem, which is formulated in $[31,32]$.

Theorem 5.1 Let $q>2$ and $\Omega$ be a bounded Lipschitz domain. Let $F \in L^{2}(\Omega)$ and $f \in L^{p}(\Omega)$ for some $2<p<q<\infty$. Suppose that for each ball $B \subset \mathbb{R}^{d}$ with the property that $|B|<c_{0}|\Omega|$, and either $4 B \subset \Omega$ or $B$ is centered on $\partial \Omega$, there exists two measurable functions $F_{B}$ and $R_{B}$ on $2 B \cap \Omega$ such that

$$
\begin{gathered}
|F| \leq\left|F_{B}\right|+\left|R_{B}\right| \text { on } 2 B \cap \Omega, \\
\left(f_{2 B \cap \Omega}\left|R_{B}\right|^{q}\right)^{1 / q} \leq C_{1}\left\{\left(f_{4 B \cap \Omega}|F|^{2}\right)^{1 / 2}+\sup _{B \subset B^{\prime} \subset 4 B_{0}}\left(f_{B^{\prime} \cap \Omega}|f|^{2}\right)^{1 / 2}\right\}, \\
\left(f_{2 B \cap \Omega}\left|F_{B}\right|^{2}\right)^{1 / 2} \leq C_{2} \sup _{B \subset B^{\prime} \subset 4 B_{0}}\left(f_{B^{\prime} \cap \Omega}|f|^{2}\right)^{1 / 2}+\delta\left(\int_{4 B \cap \Omega}|F|^{2}\right)^{1 / 2},
\end{gathered}
$$

where $C_{1}, C_{2}>0,0<c_{0}<1$. Then, there exists $\delta_{0}>0$, depending only on $C_{1}, C_{2}, c_{0}, p, q$ and the Lipschitz character of $\Omega$, such that, for any $0<\delta<\delta_{0}, F \in L^{p}(\Omega)$ and

$$
\left(f_{\Omega}|F|^{p}\right)^{1 / p} \leq C\left(f_{\Omega}|F|^{2}\right)^{1 / 2}+\left(f_{\Omega}|f|^{p}\right)^{1 / p}
$$

where $C$ depends only on $d, C_{1}, C_{2}, c_{0}, p, q$ and the Lipschitz character of $\Omega$. 
Proof of Theorem 1.2 Since the desired estimate is trivial when $p=2$, it suffices to consider the case $p>2$. Thanks to the extension theorem in [26, p. 223], for any $\dot{g}=\left\{g_{\gamma}\right\}_{|\gamma| \leq m-1} \in$ $\dot{B}_{p}^{m-1 / p}(\partial \Omega)$ there exist a $G \in W^{m, p}(\Omega)$ such that

$$
\operatorname{Tr}\left(D^{\gamma} G\right)=g_{\gamma} \quad \text { for } 0 \leq|\gamma| \leq m-1, \quad\|G\|_{W^{m, p}(\Omega)} \leq C\|\dot{g}\|_{\dot{B}_{p}^{m-1 / p}(\partial \Omega)} .
$$

Therefore, we can restrict our investigations to the problem with homogeneous boundary conditions.

$$
\mathcal{L}_{\varepsilon} \bar{u}_{\varepsilon}=\sum_{|\alpha| \leq m} D^{\alpha} \bar{f}^{\alpha} \quad \text { in } \Omega, \quad \operatorname{Tr}\left(D^{\gamma} \bar{u}_{\varepsilon}\right)=0 \quad \text { on } \partial \Omega \text { for } 0 \leq|\gamma| \leq m-1,
$$

where $\bar{u}_{\varepsilon}=u_{\varepsilon}-G$ and

$\bar{f}^{\alpha}=f^{\alpha}+(-1)^{m+1} \sum_{|\beta|=m} A^{\alpha \beta}(x / \varepsilon) D^{\beta} G$ for $|\alpha|=m, \quad$ and $\quad \bar{f}^{\alpha}=f^{\alpha} \quad$ for $|\alpha|<m$.

Let $F=\left|\nabla^{m} \bar{u}_{\varepsilon}\right|$ and $f(x)=\sum_{|\alpha| \leq m}\left|\bar{f}^{\alpha}\right|$. We only need to construct the functions $F_{B}$, $R_{B}$ and then verify the conditions (5.6), (5.7) and (5.8) to hold for balls $B\left(x_{0}, r\right)$ with the property $|B|<c_{0}|\Omega|$ and either $4 B \subset \Omega$ or $B$ is centered on $\partial \Omega$. The case of $4 B \subset \Omega$ has been investigated for interior $W^{m, p}$ estimates in [28]. So here we only consider the situation that $B$ is centered on $\partial \Omega$.

Let $B=B\left(x_{0}, r\right)$ for some $x_{0} \in \partial \Omega$ and $0<r<r_{0} / 16$. Let $v_{\varepsilon} \in H_{0}^{m}\left(4 B \cap \Omega ; \mathbb{R}^{n}\right)$ be the solution to $\mathcal{L}_{\varepsilon} v_{\varepsilon}=\sum_{|\alpha| \leq m} D^{\alpha} \bar{f}^{\alpha}$ in $4 B \cap \Omega$ and set

$$
F_{B}=\left|\nabla^{m} v_{\varepsilon}\right|, \quad R_{B}=\left|\nabla^{m} w_{\varepsilon}\right|, \quad w_{\varepsilon}=\bar{u}_{\varepsilon}-v_{\varepsilon} .
$$

Then, it is obvious that

$$
\begin{aligned}
& |F| \leq\left|F_{B}\right|+\left|R_{B}\right| \text { on } 2 B \cap \Omega, \\
& \left(f_{2 B \cap \Omega}\left|F_{B}\right|^{2}\right)^{1 / 2} \leq C\left(f_{4 B \cap \Omega}\left|\nabla^{m} v_{\varepsilon}\right|^{2}\right)^{1 / 2} \leq C\left(f_{4 B \cap \Omega}|f|^{2}\right)^{1 / 2},
\end{aligned}
$$

which imply the conditions (5.6) and (5.8). Furthermore, note that

$$
\mathcal{L}_{\varepsilon} w_{\varepsilon}=0 \text { in } 4 B \cap \Omega, \quad \operatorname{Tr}\left(D^{\gamma} w_{\varepsilon}\right)=0 \quad \text { on } 4 B \cap \partial \Omega \text { for } 0 \leq|\gamma| \leq m-1 .
$$

By Lemma 5.1, we know that for any $2<p<\infty$,

$$
\begin{aligned}
\left(f_{2 B \cap \Omega}\left|\nabla^{m} w_{\varepsilon}\right|^{p}\right)^{1 / p} & \leq C\left(f_{4 B \cap \Omega}\left|\nabla^{m} w_{\varepsilon}\right|^{2}\right)^{1 / 2} \\
& \leq C\left(f_{4 B \cap \Omega}\left|\nabla^{m} \bar{u}_{\varepsilon}\right|^{2}\right)^{1 / 2}+C\left(f_{4 B \cap \Omega}\left|\nabla^{m} v_{\varepsilon}\right|^{2}\right)^{1 / 2} \\
& \leq C\left(f_{4 B \cap \Omega}\left|\nabla^{m} \bar{u}_{\varepsilon}\right|^{2}\right)^{1 / 2}+C\left(f_{4 B \cap \Omega}|f|^{2}\right)^{1 / 2},
\end{aligned}
$$

which implies (5.7). Noticing that all the conditions in Theorem 5.1 are verified, (1.9) follows from (5.9) immediately.

Note that if $u_{\varepsilon} \in W_{0}^{m, p}\left(\Omega, \mathbb{R}^{n}\right)$ is a weak solution to $\mathcal{L}_{\varepsilon} u_{\varepsilon}=f$ in $\Omega$, and $u_{\varepsilon}^{*} \in$ $W_{0}^{m, p^{\prime}}\left(\Omega, \mathbb{R}^{n}\right)$ is a weak solution to $\mathcal{L}_{\varepsilon}^{*} u_{\varepsilon}^{*}=f^{*}$ in $\Omega$, where $p^{\prime}=p /(p-1)$. Then, we have

$$
\left\langle f, u_{\varepsilon}^{*}\right\rangle_{W^{-m, p} \times W_{0}^{m, p^{\prime}}}=\sum_{|\alpha|=|\beta|=m} \int_{\Omega} A^{\alpha \beta}(x / \varepsilon) D^{\beta} u_{\varepsilon} D^{\alpha} u_{\varepsilon}^{*}=\left\langle f^{*}, u_{\varepsilon}\right\rangle_{W^{-m, p^{\prime}} \times W_{0}^{m, p}} .
$$


Therefore, Theorem 1.2 also holds for $1<p<2$ by a standard duality argument.

As a consequence of Theorem 1.2, one can obtain $C^{m-1, \lambda}$ estimate on $u_{\varepsilon}$ in $\Omega$ immediately. However, we choose to provide a local version using the localization argument mentioned in (4.9) and (4.16) (where we did not provide any details). The result will also provide a direct comparison to Theorem 1.1 as well as Corollary 4.1 .

Corollary 5.1 Suppose that $\Omega$ is a bounded $C^{1}$ domain in $\mathbb{R}^{d}, A \in V M O\left(\mathbb{R}^{d}\right)$ satisfies (1.2)-(1.3). For any $x_{0} \in \partial \Omega$ and $0<r \leq c_{0}$, let $u_{\varepsilon} \in H^{m}\left(\Omega \cap B\left(x_{0}, 4 r\right) ; \mathbb{R}^{n}\right)$ be a weak solution to

$$
\begin{aligned}
\mathcal{L}_{\varepsilon} u_{\varepsilon} & =\sum_{|\zeta| \leq m} D^{\zeta} f^{\zeta} \text { in } \Omega \cap B\left(x_{0}, 4 r\right), \quad \operatorname{Tr}\left(D^{\gamma} u_{\varepsilon}\right)=D^{\gamma} G \text { on } \partial \Omega \cap B\left(x_{0}, 4 r\right), 0 \\
& \leq|\gamma| \leq m-1,
\end{aligned}
$$

where $f^{\zeta} \in L^{p}\left(\Omega \cap B\left(x_{0}, 4 r\right) ; \mathbb{R}^{n}\right)$ for all $|\zeta| \leq m$, and $G \in W^{m, p}\left(\Omega \cap B\left(x_{0}, 4 r\right) ; \mathbb{R}^{n}\right)$ with $p>d$ and $p \geq 2$. Then, for any $x, y \in \Omega \cap B\left(x_{0}, r\right)$,

$$
\begin{aligned}
& \left|\nabla^{m-1} u_{\varepsilon}(x)-\nabla^{m-1} u_{\varepsilon}(y)\right| \leq C \frac{|x-y|^{\lambda}}{r^{m-1+\lambda}}\left\{\left(f_{\Omega \cap B\left(x_{0}, 4 r\right)}\left|u_{\varepsilon}\right|^{2}\right)^{1 / 2}\right. \\
& \quad+r^{m-d / p}\|G\|_{W^{m, p}\left(\Omega \cap B\left(x_{0}, 4 r\right)\right)} \\
& \left.\quad+\sum_{|\zeta| \leq m} r^{2 m-|\zeta|}\left(f_{\Omega \cap B\left(x_{0}, 4 r\right)}\left|f^{\zeta}\right|^{p}\right)^{1 / p}\right\} \\
& \left\|\nabla^{k} u_{\varepsilon}\right\|_{L^{\infty}\left(\Omega \cap B\left(x_{0}, r\right)\right)} \leq C r^{-k}\left\{\left(f_{\Omega \cap B\left(x_{0}, 4 r\right)}\left|u_{\varepsilon}\right|^{2}\right)^{1 / 2}+r^{m-d / p}\|G\|_{W^{m, p}\left(\Omega \cap B\left(x_{0}, 4 r\right)\right)}\right. \\
& \left.\quad+\sum_{|\zeta| \leq m} r^{2 m-|\zeta|}\left(f_{\Omega \cap B\left(x_{0}, 4 r\right)}\left|f^{\zeta}\right|^{p}\right)^{1 / p}\right\}
\end{aligned}
$$

where $0 \leq k \leq m-1, \lambda=1-d / p$, and $C$ depends only on $d, n, m, p, \mu, \Omega$ as well as $\varrho(t)$ in (1.7).

Proof By rescaling and translation, we may assume that $r=1, x_{0}=0$. Denote $B(0, r)$ as $B_{r}$ and let $\widetilde{D}$ be a $C^{1}$ domain such that $B_{3 / 2} \cap \Omega \subset \widetilde{D} \subset B_{2} \cap \Omega$. Set $v_{\varepsilon}=u_{\varepsilon}-G$. It is obvious that $v_{\varepsilon}$ satisfies

$$
\begin{aligned}
& \mathcal{L}_{\varepsilon} v_{\varepsilon}=\sum_{|\alpha|=m} D^{\alpha}\left\{A^{\alpha \beta}(x / \varepsilon) D^{\beta} G\right\}+\sum_{|\zeta| \leq m} D^{\zeta} f^{\zeta} \quad \text { in } \Omega \cap B_{4}, \\
& \operatorname{Tr}\left(D^{\gamma} v_{\varepsilon}\right)=0 \quad \text { on } \partial \Omega \cap B_{4} \text { for } 0<|\gamma| \leq m-1 .
\end{aligned}
$$

Let $\phi \in C_{c}^{\infty}\left(B_{3 / 2}\right)$ with $\phi=1$ in $B_{1}$ and $\left|\nabla^{k} \phi\right| \leq C 2^{k}$. We have

$$
\begin{aligned}
& \mathcal{L}_{\varepsilon}\left(v_{\varepsilon} \phi\right)=\sum_{|\alpha|=|\beta|=m}\left\{D^{\alpha}\left\{A^{\alpha \beta}(x / \varepsilon) D^{\beta} G\right\} \phi\right. \\
& +\sum_{\alpha^{\prime}+\alpha^{\prime \prime}=\alpha,\left|\alpha^{\prime \prime}\right| \geq 1} C\left(\alpha^{\prime}\right) D^{\alpha^{\prime}}\left\{A^{\alpha \beta}(x / \varepsilon) D^{\beta} v_{\varepsilon}\right\} D^{\alpha^{\prime \prime}} \phi \\
& \left.+\sum_{\beta^{\prime}+\beta^{\prime \prime}=\beta,\left|\beta^{\prime \prime}\right| \geq 1} C\left(\beta^{\prime}\right) D^{\alpha}\left\{A^{\alpha \beta}(x / \varepsilon) D^{\beta^{\prime}} v_{\varepsilon} D^{\beta^{\prime \prime}} \phi\right\}\right\}+\sum_{|\zeta| \leq m} D^{\zeta} f^{\zeta} \phi \quad \text { in } \widetilde{D}, \\
& \operatorname{Tr}\left(D^{\gamma}\left(v_{\varepsilon} \phi\right)\right)=0, \quad \text { on } \partial \widetilde{D} \text { for } 0<|\gamma| \leq m-1 .
\end{aligned}
$$


Observe that for $0 \leq \ell=\left|\alpha^{\prime}\right| \leq m-1$,

$$
\begin{aligned}
& D^{\alpha^{\prime}}\left\{A^{\alpha \beta}(x / \varepsilon) D^{\beta} v_{\varepsilon}\right\} D^{\alpha^{\prime \prime}} \phi \in W^{-m, p}(\widetilde{D}) \text { if } \nabla^{m} v_{\varepsilon} \in L^{q_{\ell}}(\widetilde{D}) \text { with } q_{\ell} \\
& =\frac{d p}{(m-\ell) p+d}(<p) .
\end{aligned}
$$

Thus, we may deduce from Theorem 1.2 that

$$
\begin{aligned}
\left\|v_{\varepsilon}\right\|_{W^{m, p}\left(B_{1} \cap \Omega\right) \leq} & C\left(\int_{\widetilde{D}}\left|\nabla^{m} G\right|^{p}\right)^{1 / p}+C \sum_{0 \leq k \leq m-1}\left(\int_{\widetilde{D}}\left|\nabla^{k} v_{\varepsilon}\right|^{p}\right)^{1 / p} \\
& +C \sum_{0 \leq \ell \leq m-1}\left(\int_{\widetilde{D}}\left|\nabla^{m} v_{\varepsilon}\right|^{q_{\ell}}\right)^{1 / q_{\ell}}+C \sum_{|\zeta| \leq m}\left(\int_{\widetilde{D}}\left|f^{\zeta}\right|^{p}\right)^{1 / p} \\
\leq & C\left(\int_{B_{2} \cap \Omega}\left|\nabla^{m} G\right|^{p}\right)^{1 / p}+C \sum_{0 \leq k \leq m-1}\left(\int_{B_{2} \cap \Omega}\left|\nabla^{k} v_{\varepsilon}\right|^{p}\right)^{1 / p} \\
& +C \sum_{0 \leq \ell \leq m-1}\left(\int_{B_{2} \cap \Omega}\left|\nabla^{m} v_{\varepsilon}\right|^{q_{\ell}}\right)^{1 / q_{\ell}}+C \sum_{|\zeta| \leq m}\left(\int_{B_{2} \cap \Omega}\left|f^{\zeta}\right|^{p}\right)^{1 / p} .
\end{aligned}
$$

Let $p_{1}=d p /(d+p)$. Thanks to the Poincaré inequality and Sobolev imbedding, we have

$$
\begin{aligned}
& \left\|\nabla^{k} v_{\mathcal{E}}\right\|_{L^{p}\left(B_{2} \cap \Omega\right)} \leq C\left\|\nabla^{m} v_{\varepsilon}\right\|_{L^{p_{1}\left(B_{2} \cap \Omega\right)}} \text { for } 0 \leq k \leq m-1, \\
& \left\|\nabla^{m} v_{\varepsilon}\right\|_{L^{q_{\ell}\left(B_{2} \cap \Omega\right)}} \leq C\left\|\nabla^{m} v_{\varepsilon}\right\|_{L^{p_{1}\left(B_{2} \cap \Omega\right)}} \text { for } q_{\ell}=\frac{d p}{(m-\ell) p+d}, 0 \leq \ell \leq m-1,
\end{aligned}
$$

which, combined with (5.13), implies that

$$
\left\|v_{\varepsilon}\right\|_{W^{m, p}\left(B_{1} \cap \Omega\right)} \leq C\left\|\nabla^{m} G\right\|_{L^{p}\left(B_{2} \cap \Omega\right)}+C\left\|\nabla^{m} v_{\varepsilon}\right\|_{L^{p_{1}\left(B_{2} \cap \Omega\right)}}+C \sum_{|\zeta| \leq m}\left\|f^{\zeta}\right\|_{L^{p}\left(B_{2} \cap \Omega\right)} .
$$

If $p_{1}>2$, we can perform a bootstrap argument for finite times to obtain that

$$
\left\|v_{\varepsilon}\right\|_{W^{m, p}\left(B_{1} \cap \Omega\right)} \leq C\left\|\nabla^{m} G\right\|_{L^{p}\left(B_{3} \cap \Omega\right)}+C\left\|\nabla^{m} v_{\varepsilon}\right\|_{L^{2}\left(B_{3} \cap \Omega\right)}+C \sum_{|\zeta| \leq m}\left\|f^{\zeta}\right\|_{L^{p}\left(B_{3} \cap \Omega\right)} .
$$

By Caccioppoli's inequality, this implies that

$$
\begin{aligned}
\left\|u_{\varepsilon}\right\|_{W^{m, p}\left(B_{1} \cap \Omega\right)} & \leq C\left\{\left\|\nabla^{m} v_{\varepsilon}\right\|_{L^{2}\left(B_{3} \cap \Omega\right)}+\|G\|_{W^{m, p}\left(B_{4} \cap \Omega\right)}+\sum_{|\zeta| \leq m}\left\|f^{\zeta}\right\|_{L^{p}\left(B_{4} \cap \Omega\right)}\right\} \\
& \leq C\left\{\left\|u_{\varepsilon}\right\|_{L^{2}\left(B_{4} \cap \Omega\right)}+\|G\|_{W^{m, p}\left(B_{4} \cap \Omega\right)}+\sum_{|\zeta| \leq m}\left\|f^{\zeta}\right\|_{L^{p}\left(B_{4} \cap \Omega\right)}\right\},
\end{aligned}
$$

which gives (5.10) and (5.11) by Sobolev imbedding.

\section{Uniform $C^{m-1,1}$ estimates}

In this section, we consider uniform boundary $C^{m-1,1}$ estimates for $u_{\varepsilon}$ in $C^{1, \theta}(0<\theta<1)$ domains. Throughout the section, we always assume that $A$ satisfies (1.2) and (1.3). Similar to Sect. 4, we only need to consider equations in $\left(D_{r}, \Delta_{r}\right)$ defined as in (1.5) with the defining function $\psi \in C^{1, \theta}\left(\mathbb{R}^{d-1}\right)$ satisfying $\psi(0)=0,\|\nabla \psi\|_{C^{\theta}\left(\mathbb{R}^{d-1}\right)} \leq M_{1}$. 
Let $u_{\varepsilon} \in H^{m}\left(D_{2} ; \mathbb{R}^{n}\right)$ be a weak solution to

$$
\mathcal{L}_{\varepsilon} u_{\varepsilon}=\sum_{|\alpha| \leq m-1} D^{\alpha} f^{\alpha} \quad \text { in } D_{1}, \quad \operatorname{Tr}\left(D^{\gamma} u_{\varepsilon}\right)=D^{\gamma} G \quad \text { on } \Delta_{1} \quad \text { for } 0 \leq|\gamma| \leq m-1,
$$

where $f^{\alpha} \in L^{q}\left(D_{1} ; \mathbb{R}^{n}\right)$ with $q>d, q \geq 2$, and $G \in C^{m, \sigma}\left(D_{1} ; \mathbb{R}^{n}\right)$ for some $0<\sigma \leq \theta$. For $0<r \leq 1$, define the following auxiliary quantities,

$$
\begin{aligned}
\Phi\left(r, u_{\varepsilon}\right)= & \frac{1}{r^{m}} \inf _{P_{m-1} \in \mathfrak{P}_{m-1}}\left\{\left(f_{D_{r}}\left|u_{\varepsilon}-P_{m-1}\right|^{2}\right)^{1 / 2}+\sum_{|\alpha| \leq m-1} r^{2 m-|\alpha|}\left(f_{D_{r}}\left|f^{\alpha}\right|^{q}\right)^{1 / q}\right. \\
& \left.+\sum_{j=0}^{m} r^{j}\left\|\nabla^{j}\left(G-P_{m-1}\right)\right\|_{L^{\infty}\left(D_{r}\right)}\right\}, \\
H\left(r ; u_{\varepsilon}\right)= & \frac{1}{r^{m}} \inf _{P_{m} \in \mathfrak{P}_{m}}\left\{\left(f_{D_{r}}\left|u_{\varepsilon}-P_{m}\right|^{2}\right)^{1 / 2}+\sum_{|\alpha| \leq m-1} r^{2 m-|\alpha|}\left(f_{D_{r}}\left|f^{\alpha}\right|^{q}\right)^{1 / q}\right. \\
& \left.+\sum_{j=0}^{m} r^{j}\left\|\nabla^{j}\left(G-P_{m}\right)\right\|_{L^{\infty}\left(D_{r}\right)}+r^{m+\sigma}\left\|\nabla^{m}\left(G-P_{m}\right)\right\|_{C^{0, \sigma}\left(D_{r}\right)}\right\} .
\end{aligned}
$$

Lemma 6.1 For $0<\varepsilon \leq r \leq 1$, let $\Phi\left(r ; u_{\varepsilon}\right)$ be defined as in (6.1). Then, there exists $u_{0} \in H^{m}\left(D_{r} ; \mathbb{R}^{n}\right)$ such that $\mathcal{L}_{0} u_{0}=\sum_{|\alpha| \leq m-1} D^{\alpha} f^{\alpha}$ in $D_{r}, \operatorname{Tr}\left(D^{\gamma} u_{0}\right)=D^{\gamma} G$ on $\Delta_{r}$ for $0 \leq|\gamma| \leq m-1$, and

$$
\frac{1}{r^{m}}\left(f_{D_{r}}\left|u_{\varepsilon}-u_{0}\right|^{2}\right)^{1 / 2} \leq C\left(\frac{\varepsilon}{r}\right)^{1 / 4} \Phi\left(2 r ; u_{\varepsilon}\right),
$$

where $C$ depends only on $d, n, m, q, \sigma, \mu$ and $M$ in (1.4).

Proof The proof is the same as Lemma 4.1, and we therefore omit the details.

Lemma 6.2 Let $u_{0} \in H^{m}\left(D_{r} ; \mathbb{R}^{n}\right)$ be a weak solution to $\mathcal{L}_{0} u_{0}=\sum_{|\alpha| \leq m-1} D^{\alpha} f^{\alpha}$ in $D_{r}$ with $\operatorname{Tr}\left(D^{\gamma} u_{0}\right)=D^{\gamma} G$ on $\Delta_{r}$ for $0 \leq|\gamma| \leq m-1$. Then, there exists a $\delta \in(0,1 / 4)$, depending only on $d, n, m, q, \sigma, \mu, \theta$ and $M_{1}$ in (1.10), such that

$$
H\left(\delta r ; u_{0}\right) \leq \frac{1}{2} H\left(r ; u_{0}\right)
$$

Proof The proof, parallel to that of Lemma 4.2, is mainly based on $C^{m, \sigma}$ estimates for higherorder elliptic systems with constant coefficients in $C^{1, \theta}(0<\sigma \leq \theta)$ domains. By rescaling, we assume that $r=1$. Taking

$$
P_{m}(x)=\sum_{|\alpha| \leq m} \frac{1}{\alpha !} D^{\alpha} u_{0}(0) x^{\alpha}=\sum_{|\alpha| \leq m} \frac{1}{\alpha !} D^{\alpha} G(0) x^{\alpha},
$$

it is not difficult to find that for any $0<\delta<1 / 4$ and any $0<\sigma^{\prime}<\min \{1-d / q, \sigma\}$,

$$
\begin{aligned}
H\left(\delta, u_{0}\right) \leq & C \delta^{\sigma^{\prime}}\left\|u_{0}\right\|_{C^{m, \sigma^{\prime}\left(D_{\delta}\right)}}+C \delta^{m-|\alpha|-d / q} \sum_{|\alpha| \leq m-1}\left(f_{D_{1}}\left|f^{\alpha}\right|^{q}\right)^{1 / q} \\
& +C \delta^{\sigma}\|G\|_{C^{m, \sigma}\left(D_{1}\right)} .
\end{aligned}
$$


By the localization argument and the $C^{m, \sigma}$ estimate for higher-order elliptic systems with constant coefficients (see e.g., [27, Corollary 2.4]), we have

$$
\begin{aligned}
\left\|u_{0}\right\|_{C^{m, \sigma^{\prime}}\left(D_{\delta}\right)} & \leq C\left\|u_{0}\right\|_{C^{m, \sigma^{\prime}}\left(D_{1 / 4}\right)} \\
& \leq C\left\{\left(f_{D_{1}}\left|u_{0}\right|^{2}\right)^{1 / 2}+\sum_{|\alpha| \leq m-1}\left(f_{D_{1}}\left|f^{\alpha}\right|^{q}\right)^{1 / q}+\|G\|_{C^{m, \sigma}\left(D_{1}\right)}\right\}
\end{aligned}
$$

for $0<\sigma^{\prime}<\min \{1-d / q, \sigma\}$. Taking (6.6) into (6.5) and setting $\delta$ small enough, we get

$$
H\left(\delta, u_{0}\right) \leq \frac{1}{2}\left\{\left(f_{D_{1}}\left|u_{0}\right|^{2}\right)^{1 / 2}+\sum_{|\alpha| \leq m-1}\left(f_{D_{1}}\left|f^{\alpha}\right|^{q}\right)^{1 / q}+\|G\|_{C^{m, \sigma}\left(D_{1}\right)}\right\} .
$$

For any $P_{m} \in \mathfrak{P}_{m}$, substituting $u_{0}, G$ by $u_{0}-P_{m}$ and $G-P_{m}$, respectively, and taking the infimum, we obtain (6.4) immediately.

Lemma 6.3 Let $0<\varepsilon<1 / 2$ and $\Phi\left(r ; u_{\varepsilon}\right), H\left(r ; u_{\varepsilon}\right)$ be defined as in (6.1) and (6.2). Let $\delta$ be given by Lemma 6.2. Then, for any $r \in[\varepsilon, 1 / 2]$,

$$
H\left(\delta r ; u_{\varepsilon}\right) \leq \frac{1}{2} H\left(r ; u_{\varepsilon}\right)+C\left(\frac{\varepsilon}{r}\right)^{1 / 4} \Phi\left(2 r ; u_{\varepsilon}\right),
$$

where $C$ depends only on $d, n, m, q, \mu, \sigma, \theta$ and $M_{1}$ in (1.10).

Proof Similar to Lemma 4.3, the result follows from Lemmas 6.1 and 6.2. We thus omit the details.

Lemma 6.4 Let $H(r)$ and $h(r)$ be two nonnegative continuous functions on the interval $(0,1]$, and let $\varepsilon \in(0,1 / 4)$. Assume that

$$
\max _{r \leq t \leq 2 r} H(t) \leq C_{0} H(2 r), \quad \max _{r \leq t, s \leq 2 r}|h(t)-h(s)| \leq C_{0} H(2 r),
$$

for any $r \in[\varepsilon, 1 / 2]$, and also

$$
H(\delta r) \leq \frac{1}{2} H(r)+C_{0} \omega(\varepsilon / r)\{H(2 r)+h(2 r)\} \quad \text { for any } r \in[\varepsilon, 1 / 2],
$$

where $\delta \in(0,1 / 4)$ and $\omega$ is a nonnegative increasing function on $[0,1]$ such that $\omega(0)=0$ and $\int_{0}^{1} \omega(\varsigma) / \varsigma d \varsigma<\infty$. Then, there exists a constant $C$ depending only on $C_{0}, \delta$ and $\omega$, such that

$$
\max _{\varepsilon \leq r \leq 1}\{H(r)+h(r)\} \leq C\{H(1)+h(1)\} .
$$

Proof See Lemma 8.5 in [35].

Armed with lemmas above, we are ready to prove Theorem 1.3.

Proof of Theorem 1.3 We assume that $0<\varepsilon \leq r<1 / 4$, since if else (1.11) is just a consequence of Caccioppoli's inequality. Let $u_{\varepsilon} \in H^{m}\left(D_{1} ; \mathbb{R}^{n}\right)$ be a weak solution to

$$
\mathcal{L}_{\varepsilon} u_{\varepsilon}=\sum_{|\alpha| \leq m-1} D^{\alpha} f^{\alpha} \quad \text { in } D_{1}, \quad \operatorname{Tr}\left(D^{\gamma} u_{\varepsilon}\right)=D^{\gamma} G \text { on } \Delta_{1} \quad \text { for } 0 \leq|\gamma| \leq m-1,
$$

where $f^{\alpha} \in L^{q}\left(D_{1} ; \mathbb{R}^{n}\right)$ with $q>d, q \geq 2$, and $G \in C^{m, \sigma}\left(D_{1} ; \mathbb{R}^{n}\right)$ for some $0<\sigma \leq \theta$. For $r \in(0,1)$, let $H(r)=H\left(r, u_{\varepsilon}\right), \Phi(r)=\Phi\left(r, u_{\varepsilon}\right)$ and $\omega(y)=y^{1 / 4}$. Define

$$
h(r)=\sum_{|\alpha|=m} \frac{1}{\alpha !}\left|D^{\alpha} P_{m r}(x)\right|,
$$


where $P_{m r} \in \mathfrak{P}_{m}$ such that

$$
\begin{aligned}
H(r)=\frac{1}{r^{m}} & \left\{\left(f_{D_{r}}\left|u_{\varepsilon}-P_{m r}\right|^{2}\right)^{1 / 2}+\sum_{|\alpha| \leq m-1} r^{2 m-|\alpha|}\left(f_{D_{r}}\left|f^{\alpha}\right|^{q}\right)^{1 / q}\right. \\
& \left.+\sum_{j=0}^{m} r^{j}\left\|\nabla^{j}\left(G-P_{m r}\right)\right\|_{L^{\infty}\left(D_{r}\right)}+r^{m+\sigma}\left\|\nabla^{m}\left(G-P_{m r}\right)\right\|_{C^{0, \sigma}\left(D_{r}\right)}\right\} .
\end{aligned}
$$

Next, let us check that $H(r), h(r)$ satisfy conditions (6.8) and (6.9). From the definition, it is obvious that

$$
H(t) \leq C H(2 r) \text { for any } t \in[r, 2 r] .
$$

On the other hand, by the definition of $h(r)$,

$$
\begin{aligned}
|h(t)-h(s)| & \leq \sum_{|\alpha|=m} \frac{1}{\alpha !}\left|D^{\alpha}\left(P_{m t}-P_{m s}\right)\right|=\sum_{|\alpha|=m} \frac{1}{\alpha !}\left(f_{D_{r}}\left|D^{\alpha}\left(P_{m t}-P_{m s}\right)\right|^{2}\right)^{1 / 2} \\
& \leq C\left(f_{D_{t}}\left|\nabla^{m}\left(G-P_{m t}\right)\right|^{2}\right)^{1 / 2}+C\left(f_{D_{s}}\left|\nabla^{m}\left(G-P_{m s}\right)\right|^{2}\right)^{1 / 2} \\
& \leq C\{H(t)+H(s)\} \leq C H(2 r)
\end{aligned}
$$

where we have used the fact $r \leq t, s \leq 2 r$, the definition of $P_{m r}$ and (6.12), respectively, for the last three inequalities. Combining (6.12) with (6.13), we know that condition (6.8) is satisfied. Finally, from the definitions of $\Phi(r), H(r)$ and $h(r)$, we obtain that

$$
\begin{aligned}
\Phi(r) \leq & \frac{1}{r^{m}}\left\{\left(f_{D_{r}}\left|u_{\varepsilon}-P_{m r}\right|^{2}\right)^{1 / 2}+\sum_{|\alpha| \leq m-1} r^{2 m-|\alpha|}\left(f_{D_{r}}\left|f^{\alpha}\right|^{q}\right)^{1 / q}\right. \\
& \left.+\sum_{j=0}^{m} r^{j}\left\|\nabla^{j}\left(G-P_{m r}\right)\right\|_{L^{\infty}\left(D_{r}\right)}\right\} \\
& +\inf _{P_{m-1} \in \mathfrak{P}_{m-1}} \frac{1}{r^{m}}\left\{\left(f_{D_{r}}\left|P_{m r}-P_{m-1}\right|^{2}\right)^{1 / 2}+\sum_{j=0}^{m} r^{j}\left\|\nabla^{j}\left(P_{m r}-P_{m-1}\right)\right\|_{L^{\infty}\left(D_{r}\right)}\right\} \\
& \leq H(r)+C h(r),
\end{aligned}
$$

which, together with (6.7), implies (6.9). Note that all conditions of Lemma 6.4 are verified. Therefore, for all $\varepsilon \leq r \leq 1$,

$$
\frac{1}{r^{m}} \inf _{P_{m-1} \in \mathfrak{P}_{m-1}}\left(f_{D_{r}}\left|u_{\varepsilon}-P_{m-1}\right|^{2}\right)^{1 / 2} \leq \Phi(r) \leq C\{H(r)+h(r)\} \leq C\{H(1)+h(1)\},
$$

where $C$ depends only on $d, n, m, q, \mu, \sigma, \theta$ and $M_{1}$ in (1.10). From the definition of $H(1)$, we have

$$
\begin{aligned}
h(1) & \leq \sum_{|\alpha|=m}\left(f_{D_{1}}\left|D^{\alpha}\left(G-P_{m 1}\right)\right|^{2}\right)^{1 / 2}+C\left\|\nabla^{m} G\right\|_{L^{\infty}\left(D_{1}\right)} \\
& \leq C\left\{H(1)+\left\|\nabla^{m} G\right\|_{L^{\infty}\left(D_{1}\right)}\right\} .
\end{aligned}
$$


It then follows that

$$
\begin{aligned}
& \frac{1}{r^{m}} \inf _{P_{m-1} \in \mathfrak{P}_{m-1}}\left(f_{D_{r}}\left|u_{\varepsilon}-P_{m-1}\right|^{2}\right)^{1 / 2} \leq C\left\{\left(f_{D_{1}}\left|u_{\varepsilon}\right|^{2}\right)^{1 / 2}+\sum_{|\alpha| \leq m-1}\left(f_{D_{1}}\left|f^{\alpha}\right|^{q}\right)^{1 / q}\right. \\
&\left.+\|G\|_{C^{m, \sigma}\left(D_{1}\right)}\right\},
\end{aligned}
$$

which gives (1.11) through Caccioppoli’s inequality.

Corollary 6.1 In addition to the assumptions of Theorem 1.3, if A satisfies (1.12), then

$$
\left\|\nabla^{m} u_{\varepsilon}\right\|_{L^{\infty}\left(D_{1 / 4}\right)} \leq C\left\{\left(f_{D_{1}}\left|u_{\varepsilon}\right|^{2}\right)^{1 / 2}+\sum_{|\alpha| \leq m-1}\left(f_{D_{1}}\left|f^{\alpha}\right|^{q}\right)^{1 / q}+\|G\|_{C^{m, \sigma}\left(D_{1}\right)}\right\},
$$

where $C$ depends only on $d, n, m, q, \sigma, \mu$ as well as $\Lambda_{0}, \tau_{0}$ in (1.12) and $\theta, M_{1}$ in (1.10).

Proof It is enough to consider the case $0<\varepsilon<1 / 2$, since otherwise the coefficient is uniformly Hölder continuous and the result (6.16) is known, see e.g., [27, Corollary 2.4]. Setting

$$
v_{\varepsilon}(x)=u_{\varepsilon}(\varepsilon x)-\widetilde{G}(x), \quad \widetilde{G}(x)=G(\varepsilon x), \quad \widetilde{f}^{\alpha}(x)=\varepsilon^{2 m-|\alpha|} f(\varepsilon x),
$$

we have

$$
\left\{\begin{array}{l}
\mathcal{L}_{1} v_{\varepsilon}=\sum_{|\alpha| \leq m-1} D^{\alpha} \tilde{f}^{\alpha}(x)+\sum_{|\alpha|=|\beta|=m} D^{\alpha}\left\{A^{\alpha \beta} D^{\beta} \widetilde{G}(x)\right\} \quad \text { in } D_{1}, \\
\operatorname{Tr}\left(D^{\gamma} v_{\varepsilon}\right)=0, \quad \text { on } \Delta_{1} \text { for } 0 \leq|\gamma| \leq m-1 .
\end{array}\right.
$$

Let $\phi \in C_{c}^{\infty}\left(B_{1}\right)$ with $\phi=1$ in $B_{1 / 4}$ and $\left|\nabla^{k} \phi\right| \leq C 2^{k}$, and let $\widetilde{D}$ be a $C^{1, \theta}$ domain such that $D_{1 / 4} \subseteq \widetilde{D} \subseteq D_{1 / 2}$. We have

$$
\begin{aligned}
& \mathcal{L}_{1}\left(v_{\varepsilon} \phi\right)= E(x) \phi+\sum_{\substack{|\alpha|=|\beta|=m \\
\zeta+\eta=\beta \\
|\eta| \geq 1}} C(\zeta) D^{\alpha}\left\{A^{\alpha \beta} D^{\zeta} v_{\varepsilon} D^{\eta} \phi\right\} \\
&+\sum_{\substack{|\alpha|=|\beta|=m \\
\zeta^{\prime}+\eta^{\prime}=\alpha \\
\left|\eta^{\prime}\right| \geq 1}} C\left(\zeta^{\prime}\right) D^{\zeta^{\prime}}\left\{A^{\alpha \beta} D^{\beta} v_{\varepsilon}\right\} D^{\eta^{\prime}} \phi \text { in } \widetilde{D}, \\
& \operatorname{Tr}\left(D^{\gamma}\left(v_{\varepsilon} \phi\right)\right)=0 \quad \text { on } \partial \widetilde{D} \text { for } 0 \leq|\gamma| \leq m-1,
\end{aligned}
$$

where

$$
E(x)=\sum_{|\alpha| \leq m-1} D^{\alpha} \tilde{f}^{\alpha}(x)+\sum_{|\alpha|=|\beta|=m} D^{\alpha}\left\{A^{\alpha \beta} D^{\beta} \widetilde{G}(x)\right\} .
$$

Thanks to the boundary $C^{m, \lambda}$ estimate for operator $\mathcal{L}_{1}$ in $C^{1, \theta}$ domains [27], we know that for any $q, p>d$,

$$
\begin{aligned}
\left\|\nabla^{m} v_{\varepsilon}\right\|_{L^{\infty}\left(D_{1 / 2}\right) \leq} & C\left\{\left(f_{D_{1}}\left|v_{\varepsilon}\right|^{2}\right)^{1 / 2}+\|\widetilde{G}\|_{C^{m, \sigma}\left(D_{1}\right)}\right. \\
& \left.+\sum_{|\alpha| \leq m-1}\left(f_{D_{1}}\left|\tilde{f}^{\alpha}\right|^{q}\right)^{1 / q}+\left\|v_{\varepsilon}\right\|_{W^{m, p}(\widetilde{D})}\right\} .
\end{aligned}
$$


Thanks to the $W^{m, p}$ estimate for (6.17), there exists some $p>d$ such that

$$
\left\|v_{\varepsilon}\right\|_{W^{m, p}(\widetilde{D})} \leq C\left\{\left(f_{D_{1}}\left|v_{\varepsilon}\right|^{2}\right)^{1 / 2}+\sum_{|\alpha| \leq m-1}\left(f_{D_{1}}\left|\widetilde{f}^{\alpha}\right|^{q}\right)^{1 / q}+\|\widetilde{G}\|_{C^{m, \sigma}\left(D_{1}\right)}\right\},
$$

which, combined with (6.18), implies that

$$
\left\|\nabla^{m} v_{\varepsilon}\right\|_{L^{\infty}\left(D_{1 / 2}\right)} \leq C\left\{\left(f_{D_{1}}\left|v_{\varepsilon}\right|^{2}\right)^{1 / 2}+\sum_{|\alpha| \leq m-1}\left(f_{D_{1}}\left|\tilde{f}^{\alpha}\right|^{q}\right)^{1 / q}+\|\widetilde{G}\|_{C^{m, \sigma}\left(D_{1}\right)}\right\} .
$$

It then follows from the change of variables that

$$
\begin{aligned}
\left\|\nabla^{m} u_{\varepsilon}\right\|_{L^{\infty}\left(D_{\varepsilon / 2}\right)} \leq & C \frac{1}{\varepsilon^{m}}\left\{\left(f_{D_{\varepsilon}}\left|u_{\varepsilon}\right|^{2}\right)^{1 / 2}+\varepsilon^{2 m-|\alpha|} \sum_{|\alpha| \leq m-1}\left(f_{D_{\varepsilon}}\left|f^{\alpha}\right|^{q}\right)^{1 / q}\right. \\
& \left.+\sum_{j=0}^{m} \varepsilon^{j}\left\|\nabla^{j} G\right\|_{L^{\infty}\left(D_{\varepsilon}\right)}+\varepsilon^{m+\sigma}\left\|\nabla^{m} G\right\|_{C^{0, \sigma}\left(D_{\varepsilon}\right)}\right\} .
\end{aligned}
$$

Using (6.11), (6.14) and (6.15), we may conclude from (6.19) that

$$
\begin{aligned}
\left\|\nabla^{m} u_{\varepsilon}\right\|_{L^{\infty}\left(D_{\varepsilon / 2}\right)} & \leq C\{H(\varepsilon)+h(\varepsilon)\} \leq C\{H(1)+h(1)\} \\
& \leq C\left\{\left(f_{D_{1}}\left|u_{\varepsilon}\right|^{2}\right)^{1 / 2}+\sum_{|\alpha| \leq m-1}\left(f_{D_{1}}\left|f^{\alpha}\right|^{q}\right)^{1 / q}+\|G\|_{C^{m, \sigma}\left(D_{1}\right)}\right\} .
\end{aligned}
$$

This, together with the interior uniform $C^{m-1,1}$ estimate for $u_{\varepsilon}$ derived in [28, Theorem 1.2], gives (6.16).

Acknowledgements This paper was completed during the authors' visits at the University of Kentucky. We are much obliged to Professor Zhongwei Shen for the guidance and hospitality. Our special thanks also go to Professor Russell Brown and the Department of Mathematics for the warm hospitality and support, as well as to Jinping Zhuge for some enlightening discussions.

\section{References}

1. Agranovich, M.S.: On the theory of Dirichlet and Neumann problems for linear strongly elliptic systems with Lipschitz domains. Funktsional. Anal. i Prilozhen. 41(4), 1-21 (2007). 96

2. Armstrong, S.N., Mourrat, J.C.: Lipschitz regularity for elliptic equations with random coefficients. Arch. Ration. Mech. Anal. 219(1), 255-348 (2016)

3. Armstrong, S.N., Shen, Z.: Lipschitz estimates in almost-periodic homogenization. Commun. Pure Appl. Math. 69(10), 1882-1923 (2016)

4. Armstrong, S.N., Smart, C.K.: Quantitative stochastic homogenization of convex integral functionals. Ann. Sci. Éc. Norm. Supér. (4) 49(2), 423-481 (2016)

5. Avellaneda, M., Lin, F.: Compactness methods in the theory of homogenization. Commun. Pure Appl. Math. 40(6), 803-847 (1987)

6. Avellaneda, M., Lin, F.: Compactness methods in the theory of homogenization. II. Equations in nondivergence form. Commun. Pure Appl. Math. 42(2), 139-172 (1989)

7. Avellaneda, M., Lin, F.: $L^{p}$ bounds on singular integrals in homogenization. Commun. Pure Appl. Math. 44(8-9), 897-910 (1991)

8. Barton, A.: Gradient estimates and the fundamental solution for higher-order elliptic systems with rough coefficients. Manuscr. Math. 151(3-4), 375-418 (2016)

9. Bensoussan, A., Lions, J.L., Papanicolaou, G.: Asymptotic Analysis for Periodic Structures, vol. 5. NorthHolland Publishing Company, Amsterdam (1978)

10. Byun, S., Ryu, S.: Gradient estimates for higher order elliptic equations on nonsmooth domains. J. Differ. Equ. 250(1), 243-263 (2011) 
11. Caffarelli, L.A., Peral, I.: On $W^{1, p}$ estimates for elliptic equations in divergence form. Commun. Pure Appl. Math. 51(1), 1-21 (1998)

12. Dong, H., Kim, D.: Higher order elliptic and parabolic systems with variably partially BMO coefficients in regular and irregular domains. J. Funct. Anal. 261(11), 3279-3327 (2011)

13. Dong, H., Kim, D.: On the $L_{p}$-solvability of higher order parabolic and elliptic systems with BMO coefficients. Arch. Ration. Mech. Anal. 199(3), 889-941 (2011)

14. Geng, J.: $W^{1, p}$ estimates for elliptic problems with Neumann boundary conditions in Lipschitz domains. Adv. Math. 229(4), 2427-2448 (2012)

15. Geng, J., Shen, Z., Song, L.: Uniform $W^{1, p}$ estimates for systems of linear elasticity in a periodic medium. J. Funct. Anal. 262(4), 1742-1758 (2012)

16. Gloria, A., Neukamm, S., Otto, F.: A regularity theory for random elliptic operators (2014). arXiv preprint arXiv: 1409.2678

17. Gloria, A., Neukamm, S., Otto, F.: Quantification of ergodicity in stochastic homogenization: optimal bounds via spectral gap on Glauber dynamics. Invent. Math. 199(2), 455-515 (2015)

18. Griso, G.: Error estimate and unfolding for periodic homogenization. Asymptot. Anal. 40(3-4), 269-286 (2004)

19. Grisvard, P.: Elliptic Problems in Nonsmooth Domains (Classics in Applied Mathematics), vol. 69. SIAM, Philadelphia (2011)

20. Gu, S., Shen, Z.: Homogenization of Stokes systems and uniform regularity estimates. SIAM J. Math. Anal. 47(5), 4025-4057 (2015)

21. Hofmann, S., Kenig, C., Mayboroda, S., Pipher, J.: Square function/non-tangential maximal function estimates and the dirichlet problem for non-symmetric elliptic operators. J. Am. Math. Soc. 28, 483-529 (2015)

22. Kenig, C.E., Lin, F., Shen, Z.: Convergence rates in $L^{2}$ for elliptic homogenization problems. Arch. Ration. Mech. Anal. 203(3), 1009-1036 (2012)

23. Kenig, C.E., Lin, F., Shen, Z.: Homogenization of elliptic systems with Neumann boundary conditions. J. Am. Math. Soc. 26(4), 901-937 (2013)

24. Kenig, C.E., Prange, C.: Uniform Lipschitz estimates in bumpy half-spaces. Arch. Ration. Mech. Anal. 216(3), 703-765 (2015)

25. Kukushkin, A.A., Suslina, T.A.: Homogenization of high-order elliptic operators with periodic coefficients. Algebra i Analiz 28(1), 89-149 (2016)

26. Maz'ya, V., Mitrea, M., Shaposhnikova, T.: The Dirichlet problem in Lipschitz domains for higher order elliptic systems with rough coefficients. J. Anal. Math. 110, 167-239 (2010)

27. Miyazaki, Y.: Schauder theory for Dirichlet elliptic operators in divergence form. J. Evol. Equ. 13(2), 443-480 (2013)

28. Niu, W., Shen, Z., Xu, Y.: Convergence rates and interior estimates in homogenization of higher order elliptic systems. J. Funct. Anal. 274(8), 2356-2398 (2018)

29. Pastukhova, S.E.: Estimates in homogenization of higher-order elliptic operators. Appl. Anal. 95(7), 1449-1466 (2016)

30. Pastukhova, S.E.: Operator error estimates for homogenization of fourth order elliptic equations. St. Petersburg Math. J. 28(2), 273-289 (2017)

31. Shen, Z.: Bounds of Riesz transforms on $L^{p}$ spaces for second order elliptic operators. Ann. Inst. Fourier 55(1), 173-197 (2005)

32. Shen, Z.: The $L^{p}$ boundary value problems on Lipschitz domains. Adv. Math. 216(1), 212-254 (2007)

33. Shen, Z.: $W^{1, p}$ estimates for elliptic homogenization problems in nonsmooth domains. Indiana Univ. Math. J. 57(5), 2283-2298 (2008)

34. Shen, Z.: Convergence rates and Hölder estimates in almost-periodic homogenization of elliptic systems. Anal. PDE 8(7), 1565-1601 (2015)

35. Shen, Z.: Boundary estimates in elliptic homogenization. Anal. PDE 10(3), 653-694 (2017)

36. Shen, Z., Zhuge, J.: Approximate correctors and convergence rates in almost-periodic homogenization. J. Math. Pures Appl. (9) 110, 187-238 (2018)

37. Suslina, T.A.: Homogenization of the Dirichlet problem for elliptic systems: $L^{2}$-operator error estimates. Mathematika 59(2), 463-476 (2013)

38. Suslina, T.A.: Homogenization of the Neumann problem for elliptic systems with periodic coefficients. SIAM J. Math. Anal. 45(6), 3453-3493 (2013)

39. Suslina, T.A.: Homogenization of the dirichlet problem for higher-order elliptic equations with periodic coefficients. Algebra i Analiz 29, 139-192 (2017)

40. Suslina, T.A.: Homogenization of the Neumann problem for higher-order elliptic equations with periodic coefficients (2017, preprint). arXiv: 1705.08295 
41. Verchota, G.C.: Potentials for the Dirichlet Problem in Lipschitz Domains, Potential theory-ICPT 94 (Kouty, 1994), de Gruyter, Berlin, pp. 167-187 (1996)

42. $\mathrm{Xu}, \mathrm{Q} .:$ Convergence rates for general elliptic homogenization problems in Lipschitz domains. SIAM J. Math. Anal. 48(6), 3742-3788 (2016)

43. Xu, Q.: Uniform regularity estimates in homogenization theory of elliptic systems with lower order terms on the Neumann boundary problem. J. Differ. Equ. 261(8), 4368-4423 (2016)

44. $\mathrm{Xu}, \mathrm{Q} .:$ Convergence rates and $W^{1, p}$ estimates in homogenization theory of Stokes systems in Lipschitz domains. J. Differ. Equ. 263(1), 398-450 (2017)

45. Xu, Y., Niu, W.: Convergence rates in almost-periodic homogenization of higher-order elliptic systems (2017). arXiv preprint arXiv:1712.01744

46. Zhuge, J.: Uniform boundary regularity in almost-periodic homogenization. J. Differ. Equ. 262(1), 418453 (2017) 